Tidal effects in stars, planets and disks

M.-J. Goupil and J.-P. Zahn

EAS Publications Series, Vol. ?, 2018

\title{
OBSERVATIONAL EVIDENCE FOR TIDAL INTERACTION IN CLOSE BINARY SYSTEMS
}

\author{
Tsevi Mazeh ${ }^{1}$
}

\begin{abstract}
This paper reviews the rich corpus of observational evidence for tidal effects, mostly based on photometric and radial-velocity measurements. This is done in a period when the study of binaries is being revolutionized by large-scaled photometric surveys that are detecting many thousands of new binaries and tens of extrasolar planets.

We begin by examining the short-term effects, such as ellipsoidal variability and apsidal motion. We next turn to the long-term effects, of which circularization was studied the most: a transition period between circular and eccentric orbits has been derived for eight coeval samples of binaries. The study of synchronization and spin-orbit alignment is less advanced. As binaries are supposed to reach synchronization before circularization, one can expect finding eccentric binaries in pseudo-synchronization state, the evidence for which is reviewed. We also discuss synchronization in PMS and young stars, and compare the emerging timescale with the circularization timescale.

We next examine the tidal interaction in close binaries that are orbited by a third distant companion, and review the effect of pumping the binary eccentricity by the third star. We elaborate on the impact of the pumped eccentricity on the tidal evolution of close binaries residing in triple systems, which may shrink the binary separation.

Finally we consider the extrasolar planets and the observational evidence for tidal interaction with their parent stars. This includes a mechanism that can induce radial drift of short-period planets, either inward or outward, depending on the planetary radial position relative to the corotation radius. Another effect is the circularization of planetary orbits, the evidence for which can be found in eccentricityversus-period plot of the planets already known.

Whenever possible, the paper attempts to address the possible confrontation between theory and observations, and to point out noteworthy cases and observations that can be performed in the future and may shed some light on the key questions that remain open.
\end{abstract}

${ }^{1}$ School of Physics of Astronomy, Sackler Faculty of Exact Sciences, Tel Aviv University

(c) EDP Sciences 2018

DOI: (will be inserted later) 
Tidal effects in stars, planets and disks

\section{Introduction}

Stars in close binary systems are subject to mutual tidal forces that distort their stellar shape, breaking their spherical and axial symmetry, which leads to different observational effects. This paper reviews the rich corpus of observational evidence for the tidal effects, which has been accumulated mostly by photometric and radialvelocity studies of short-period binaries.

One can divide the observational effects of the stellar distortion caused by tidal interaction into three classes:

- Direct observations of the distorted shape of the two components of the binary system. One example is the ellipsoidal periodic modulation of the photometric intensity of the binary with the orbital period, caused by the rotation of the stars with the ellipsoidal shape.

- Deviation of the orbital motion of the binary from pure Keplerian orbit. This is caused by departures of the gravitational attraction from the inversesquare law, due to the distortion of the two stars from spherical symmetry. One example is the famous apsidal motion observed in many eccentric eclipsing binaries.

- Long timescale evolution of the binary orbital elements and the stellar rotation, induced by the tidal interaction. The long-term interaction will take place as long as the tides raised by the mutual interaction vary in size, shape and location on the surface of the two components. The tides do not vary only when

- the binary motion is circularized,

- the stellar rotation is synchronized with the binary orbital motion, and

- the stellar rotation axis is aligned with the normal to the orbital plane of motion.

Observational evidence for this long-term evolution can be found, for example, through the circular orbits of short-period binaries.

The timescales of the three classes are substantially different. The ellipsoidal effect varies with the orbital period, which is of the order of a few days. The timescale of the deviations of the binary motion from the Keplerian orbit is in the range between tens and thousands of years, whereas the last class timescale is of the order of millions or billions of years. Therefore, we can witness close binaries for which the effects of the first and the second class are in action, while observation of the third class of effects can be made only retroactively, in recognition of the results of a long timescale processes.

Observing one or more of these effects allows us to learn about the response of each star to the tidal forces exerted by its companion. This response depends on the stellar internal structure, and therefore observations of these effects can 
provide us with a unique opportunity to study that structure in a manner which is not possible with single stars.

In the last few decades numerous observations of the tidal interaction in close binaries have been accumulated. In many cases, observations of binaries performed for other reasons could not have been accounted for without the tidal effects. One example is the lightcurves of eclipsing binaries, which are usually observed in order to derive the geometrical elements of the systems. However, the lightcurves of these close systems can not be fully understood without taking into account the distortion of the two stars. In other cases, special observational effort has been made in order to detect some evidence for tidal effects. One example is the search for the circularization cutoff period in samples of coeval binaries. Both types of cases give us an opportunity to confront the tidal theory, based on stellar structure and evolution, with the observations. This paper will review both types of observations.

Sections $2 \& 3$ review the observational evidence for the ellipsoidal modulation and the apsidal motion of eccentric binaries. Section 4, 5, and 6 discuss the evidence for long-term circularization, synchronization and alignment of shortperiod binaries. Section 7 covers the tidal interaction in triple systems, when the third distant companion can have an impact on the tidal evolution of the close binary.

The study of tidal interaction between the recently discovered extrasolar planets and their parent stars has gained rapid momentum since 1995, following the discovery of the first confirmed extrasolar planet. Section 8 reviews some aspects of this fascinating new field.

This paper has been written at a juncture when the study of close binaries has been undergoing a transitional phase, the result of large-scaled photometric surveys which are starting to produce large samples of eclipsing binaries. In acknowledgment of the new era, Section 9 reviews some of the recent results from two of these surveys. Finally, Section 10 summarizes the previous sections and discusses some aspects of future studies of tidal interaction in close binaries.

This paper is too short and my knowledge too incomplete to cover all the studies in the different aspects of the subject. I made a great effort, though, to cover in each of the sections at least the open questions in that subfield. I apologize to those whose work was not included in this review. I have made an effort to keep the notation of each section consistent with the other sections of this review. This forced me to adopt some notations that are not so common in the different fields covered by this paper. Each section was written as a separate piece of study, so many definitions were repeated throughout this paper.

Finally, I hope that this review will be of some use to young researchers in the field. It would fulfill my highest expectations if some research is initiated as a result of reading this review and considering some of questions that it raises. 
Tidal effects in stars, planets and disks

\section{Ellipsoidal effect}

Ellipsoidal variables are close binary systems whose lightcurves show periodic variations due to the distortion of the components caused by their mutual attraction. Usually, this term is used (e.g., Morris \& Naftilan 1993) to denote binaries whose orbital inclinations are not large enough to render them eclipsing systems, although the effect is seen in eclipsing binaries as well.

When calculating the ellipsoidal effect (e.g., Kopal 1959, Morris 1985, Morris \& Naftilan 1993) one usually assumes that the two stars are in equilibrium in the binary rotating reference frame, which means that the stars are synchronized and aligned and that the orbit is circularized. Models of stellar evolution and circularization and synchronization (e.g., Zahn 1977) have shown that for binaries with periods less than a few days these conditions are reached on a much shorter timescale than the lifetimes of the stars. Extensive observational evidence supports this theoretical result (see Sections 4-6). For longer periods, the ellipsoidal effect is small anyway.

To derive the ellipsoidal effect, Morris \& Naftilan (1993) expanded the periodic variation into discrete Fourier series with terms that depend on the ratio $R_{*} / a$, where $R_{*}$ is the stellar radius and $a$ is the semi-major axis of the binary orbit. Assuming $R_{*} / a$ is small, the leading term to present the variation of the primary, with a radius $R_{1}$, of the order of $\left(R_{1} / a\right)^{3}$, has a semi-amplitude of:

$$
\left(\frac{\Delta F_{1}}{F_{1}}\right)_{\text {ellips }}=0.15 \frac{\left(15+u_{1}\right)\left(1+g_{1}\right)}{3-u_{1}} \frac{M_{2}}{M_{1}}\left(\frac{R_{1}}{a}\right)^{3} \sin ^{2} i .
$$

In the above expression, $g_{1}$ is the gravity darkening coefficient of the primary and $u_{1}$ is its limb-darkening coefficient, and $i$ is the orbital inclination.

For a period of a few days and in main-sequence stars, the amplitude of the ellipsoidal effect is a few percent, and therefore the effect can be easily measured. For a spectroscopic binary, known by its radial-velocity modulation, measuring the amplitude of the ellipsoidal effect can substantially add to our knowledge of the system, as this amplitude can add some constraints on the stellar radius, mass and the binary inclination. If we can estimate the stellar radius from its spectral type and the semi-major axis of the binary from its period, we can estimate the inclination of the binary, which is a crucial parameter for accurately obtaining the stellar masses of double-lined spectroscopic binaries.

Observational astronomy is always complex, and the ellipsoidal effect is no exception. First, when calculating the expected amplitude of the effect, one must take into account the variation of the secondary star as well. In fact, as the intensities of the two stars are both modulated with half the orbital period, the observed effect is the weighted average of the two stars. Therefore, deriving the inclination from the ellipsoidal amplitude is not a trivial matter and might necessitate observations in a few wavelengths. Second, other stellar modulations might also be present, like in the case of the $\delta$ Scuti star XX Pyx (Aerts et al. 2002). Fortunately, the ellipsoidal effect has a clear periodicity of half the orbital period, 
which is different, for example, from the stellar spot modulation that varies, for a synchronized system, with the orbital period.

\subsection{Interesting systems: Black holes in X-ray binaries}

One field in which the ellipsoidal variation was found to be critical is the study of X-ray binaries, which are close binaries with a compact object - a white dwarf, a neutron star or a black hole, together with a main-sequence or a giant optical companion. The X-ray luminosity, generated around the compact object, is fuelled by mass transfer from the companion onto the compact object. In many of these systems, the companion fills its Roche lobe and therefore its shape is substantially distorted and the corresponding ellipsoidal effect is relatively large.

In almost all the X-ray binaries, the ellipsoidal effect is crucial to putting strong constraints on the orbital inclination and the mass of the compact object. The mass of the compact object is the only way to identify stellar black holes $(=\mathrm{BH})$, and therefore the ellipsoidal variability is an essential step in studying these general-relativity objects (e.g., Orosz 2003). Although one has to take into account the unknown contribution of the accretion disc to the luminosity of the system, careful analysis can give some constraints on the mass of the compact object (e.g., Charles \& Coe 2006). Two such examples are V616 Mon (e.g., Harrison et al. 2007) and XTE J118+480 (Gelino et al. 2006).

An interesting case is M33 X-7, the first stellar BH found in the neighbouring galaxy M33 (Pietsch et al. 2006). With a period of 3.45 days, the ellipsoidal amplitude was measured to be of about 4\% (Shporer et al. 2007), and contributed to the derivation of a mass of $15.65 \pm 1.45 M_{\odot}$ (Orosz et al. 2007).

\section{Apsidal motion}

Apsidal motion, the precession of the orbital ellipse in the binary plane of motion, can be measured by the detection of a small change of $\omega$ - the angle denoting the direction of the line of apsides in the orbital plane. In a Keplerian motion the ellipse and its orientation are constants of motion, and only deviation from Newtonian inverse-square law attraction can cause the ellipse to precess. The deviation results from the asymmetrical stellar shape, caused by the tidal forces of its companion. The precession period is longer than the orbital period by many orders, and therefore one can first derive the osculating orbital parameters, ignoring the apsidal precession, and only afterward search for variations of $\omega$ over a longer timescale.

The classical theoretical derivation of the precession rate ignores the general relativity effect and assumes spin-orbit alignment of the two stars (see discussion of the two effects in Subsections 3.1 and 3.2). To calculate theoretically the expected rate of apsidal motion one has first to derive the stellar shape induced by the tides, and then to calculate the resulting advance of the line of apsides. For an historical review of the theoretical calculation which started back in 1928 by Russell (1928), see Claret \& Giménez (1993). The apsidal precession period due 
only to the primary star, $U_{\text {tidal, } 1}$, taking into account only the linear part of the stellar quadruple moment and up to the second order in the eccentricity $e$, is given by the formula:

$$
\frac{P}{U_{\text {tidal }, 1}} \simeq k_{2,1}\left(\frac{R_{1}}{a}\right)^{5}\left[15 f_{2}(e) q+\left(\frac{\Omega_{1, \text { rot }}}{n_{\text {orbit }}}\right)^{2}(1+q)\right]
$$

where $P$ is the orbital period, $q$ is the mass ratio $-M_{2} / M_{1}$, where $M_{1}$ is the primary mass and $M_{2}$ is the secondary mass, $\Omega_{1, \text { rot }}$ is the rotational frequency of the primary, $n_{\text {orbit }}=2 \pi / P$ is the averaged orbital frequency, $R_{1}$ is the radius of the primary, $a$ is the orbital semi-major axis, and $f_{2}(e) \simeq\left(1+\frac{3}{2} e^{2}\right)$ is a function of $e$ of order unity. The parameter $k_{2,1}$ is the second-order coefficient of the internal structure of the primary, and reflects the stellar radial concentration (e.g., Cowling 1938; Sterne 1939a; b; c; Kopal 1959). For infinite degree of concentration of the primary (mass point) $k_{2,1}$ becomes zero, while for a homogeneous configuration $k_{2,1}=0.75$ (e.g., Claret \& Giménez 1993). Typical models yield $k_{2,1}$ of the order of $0.01-0.001$, depending on the stellar mass and age. The precession is in the direction of the orbital motion, and therefore $\dot{\omega}$ is always positive. Such a precession is sometimes termed direct precession.

In most systems one must also take into account the secondary contribution to the precession, and therefore one uses $\bar{k}_{2}$ - some weighted average of $k_{2,1}$ and $k_{2,2}$, the latter being the structure parameter of the secondary (e.g., Claret 1999).

Apsidal motion can be measured with relative accuracy in eccentric eclipsing binaries, as $e$ and $\omega$ determine the time interval between the primary and secondary minima (e.g., Guinan \& Maloney 1985). When the time interval is substantially different from half the orbital period, small changes of $\omega$ can be detected. Observational determination of timing of minima, performed over a baseline of the order of ten years, can reveal a precession of a degree or two, indicating a precession period of thousands of years.

For double-lined spectroscopic eclipsing binaries, where the masses and radii of the two stars can be derived from the lightcurve and the radial-velocity curves, observation of the apsidal precession period can directly tell us the value of $\bar{k}_{2}$. Therefore, the apsidal motion is a way to confront the theory of stellar structure with observations (e.g., Giménez et al. 1987; Giménez 1990). There are very few contemporary observations that can directly challenge the stellar structure theory without relying on stellar evolution. Solar neutrino experiment (e.g., Bahcall \& Ulrich 1988) and stellar seismology (e.g., Dziembowski \& Pamyatnykh 1991) are two of the very few other examples. This is why the apsidal motion, although limited to eclipsing binaries with short periods, is of fundamental importance to stellar astrophysics. For a compilation of the binaries with measured apsidal motion up to their time see Petrova \& Orlov (1999). Very recently Bulut \& Demircan (2007) published a new catalog of 124 eclipsing binaries, including the pertinent observed apsidal motions.

Claret \& Giménez (1993; see also Claret 1999; 2007) carefully considered the observational result of 14 double-lined eclipsing binaries with known absolute di- 
mensions and accurately observed apsidal motion rates. They also included in their study an additional ten systems with lower quality data. Although there have been arguments for some inconsistencies between the old stellar models and the observed apsidal motion, Claret \& Giménez found that the observations are consistent with their modern stellar models (see also Claret \& Willems 2002 and Petrova \& Orlov 2002; 2003). As in the case of the solar neutrino (Bahcall et al. 2001), the consistency between the theory and observation of the apsidal motion for most of the binaries should be considered one of the great achievements of stellar astrophysics.

Apsidal motion can still refine our understanding of stellar interiors. This is especially true of stars with convective cores, where the present models include a somewhat arbitrarily chosen value of the overshooting parameter (e.g., Claret $\&$ Willems 2002). The value of this parameter can be deduced from the apsidal motion precession period, as was done in the study of V380 Cyg (Guinan et al. 2000), which consists of two B stars.

Given the agreement between theory of stellar structure and observations, we can now reverse the reasoning and use the observed tidal apsidal precession to derive the masses of the systems for spectroscopic binaries that do not show any eclipse (Jeffery 1984). For those systems, detection of the tidal precession can provide missing information about the orbital inclination, information that can be found in eclipsing binaries from the depth, shape and duration of the eclipse. Although the measurement of the precession in spectroscopic binaries is more difficult than in eclipsing binaries, Benevenuto et al. (2002) used this method to derive the masses of the two O-stars in the HD 93205 binary system.

\subsection{Relativistic Precession}

Deviation from inverse-square law gravitational attraction in binary systems appears also because of a general relativistic $(=\mathrm{GR})$ effect that in some cases can not be neglected. The resulting direct apsidal precession amounts to

$$
U_{G R}=1800\left(1-e^{2}\right)\left(\frac{P}{\text { day }}\right)^{5 / 3}\left(\frac{M_{1}+M_{2}}{M_{\odot}}\right)^{-2 / 3} \text { yrs . }
$$

(e.g., Weinberg 1972). The combined precession period, $U_{t o t}$, is

$$
\frac{1}{U_{t o t}}=\frac{1}{U_{G R}}+\frac{1}{U_{\text {tidal }}} .
$$

Therefore, in order to derive the tidal precession from any observed precession we have, in principle, to subtract the GR effect. Fortunately, the GR precession can be predicted accurately, as it depends only on orbital parameters and does not depend on the stellar radius and internal structure.

In order to compare the two precessions, we note that although for constant $R_{1} / a$ the apsidal motion goes linearly with $P$, for constant primary stellar radius $R_{1}$ we get that 


$$
\frac{1}{U_{\text {tidal }}} \propto P^{-13 / 3} \text { while } \frac{1}{U_{G R}} \propto P^{-5 / 3} .
$$

Therefore, for binaries with long enough periods, the tidal precession becomes dominant and the GR precession can be neglected (see Figure 8 and the relevant discussion).

\subsection{The effect of spin-orbit misalignment on the precession rate}

Another effect that can change the apsidal precession rate is spin-orbit misalignment of one or two of the stars in the binary system. One possible example for this effect is DI Her, which is one of the eclipsing binaries that has presented a persistent challenge to the theory of apsidal precession (Martinov \& Khaliullin 1980) for more than 25 years. The system consists of two bright B stars orbiting each other with a period of 10.55 days and eccentricity of $e=0.49$. For an historic account of the study of the system see Guinan \& Maloney (1985, hereafter GM85).

Modern value for the apsidal motion of DI Her was observed (GM85) to be 55, 400 yrs. However, the stellar models, which rely on the observed stellar radii and rotations, yielded a much shorter tidal precession period - of the order of 20,000 yrs. The inconsistency with the theory got worse, as the GR precession period, which does not depend on any stellar model, was estimated to be even shorter - 15, 400 yrs. Together, the two effects amounted to a precession period as short as 8,400 yrs, almost one order of magnitude shorter than the observed apsidal period. Such a confrontation between theory and observations can be very fruitful. It can indicate either that the theory is wrong, or that we are ignoring effects that are important for the system.

Shakura (1985; see also Company et al. 1988) suggested one possible way to 'save the phenomenon' of DI Her, by assuming the rotation axis of at least one of the two stars is not aligned with the orbital angular momentum of the system. In such a case, the companion's gravitational force exerted on the equatorial stellar rotational bulge induces a counter precession of the orbit, the rate of which depends on the angle of misalignment and the size of the equatorial bulge. The net result of the misalignment could be a substantial reduction of the apsidal precession rate. Reisenberger \& Guinan (1989), who studied Shakura's idea in depth, concluded that in order to account for the observed slow precession of DI Her one has to assume a misalignment of about $50^{\circ}$.

DI Her is not the only binary for which the apsidal motion might indicate some spin-orbit misalignment. Petrova \& Orlov (2003) discussed the possible misalignment effect in broader context, and considered a few more interesting systems.

The alignment of the rotational axes of the stars with the angular momentum of their binary orbit is discussed in detail in Section 6. The conjecture that the stellar spin axes are not aligned with the binary motion involves two interesting theoretical assumptions. First, if this idea is true for DI Her and the other systems, it means that these binaries were formed with misaligned stellar rotation. Second, 
the present misalignment means that the long-term tidal dissipation processes did not have enough time to align the stellar spins (see Section 6 below). In fact, Reisenberger \& Guinan (1989) estimated that the lifetime of the two components of DI Her is substantially shorter than the alignment timescale, and therefore one would expect DI Her to retain its original spin-orbit misalignment.

Spin-orbit misalignment can cause two additional interesting phenomena, in addition to the counter apsidal precession. The binary interaction causes the stellar rotation axis and the orbital orbit to precess around the total angular momentum of the system with the same period. Such a forced precession will change the stellar spin relative to our line of sight, resulting in a change of the stellar rotational broadening of the two stars. It might also change slightly the inclination angle of the binary, which modifies the eclipse parameters. Reisenberger \& Guinan (1989) followed Shakura's suggestion and tried to find observational hints for these two modulations, but the evidence they have collected was inconclusive. Since almost 20 years have passed since the study of Reisenberger \& Guinan, an observational revisit of the system might yield more reliable evidence that can either confirm or refute the orbital and stellar precession, as the time baseline of the inclination measurements will be then quite longer.

We note that the advance of the observational techniques since the mid-1980's renders the Shakura conjecture for DI Her easily observable. During the eclipse one can observe the Rossiter-McLaughlin effect (Rossiter 1924; McLaughlin 1924 - see Section 6.1 for an extensive discussion), by which the stellar absorption lines display systematic deviation from their normal rotational broadening profile, because some parts of the stellar surface are being eclipsed. As Section 6.2.2 points out, such observations were recently obtained for V1143 Cyg (Albrecht et al. 2007). It would be of great interest to confirm Shakura's idea, particularly because of its implications for binary formation, as well as because competing ideas to solve the mystery of DI Her have been discussed in the literature (Claret 1998).

\section{Circularization}

We move now to consider the observational evidence for long-term dissipative processes taking place in short-period binaries. This section will consider the evidence for circularization, while the next two sections will review the evidence for synchronization and alignment.

It is not easy to find observational evidence for the circularization processes, as we are not able to follow any binary that goes through the long-term circularization. Instead, we can only observe circular binaries that we guess were formed with eccentric orbits. However, like every retrospect line of evidence, such observations are open to other interpretations, and the conclusions are not unquestionable.

On the theoretical side, the calculations of the circularization processes and their effects are more complicated than those of the ellipsoidal modulation and the precession period. This is so because in the latter two processes one assumes an immediate stellar response to the tidal forces, while the derivation of circularization timescale involves a response lag between the tidal forces and the stellar shape 
(e.g., Zahn 1966). As we shall see, this entails serious disagreement between the theoreticians, which makes the observational evidence even more important.

To first approximation, the tidal interaction between the two components of a binary reduces the orbital eccentricity $e$ such that

$$
\frac{d e}{d t}=-C e,
$$

where the factor $C$ depends on the orbital separation, the internal structure of the two stars and their rotation. As $C$ varies very slowly, usually on the timescale of the stellar lifetime, we can assume in many cases that $C$ is constant. Therefore, the eccentricity decays exponentially, and we can define the circularization timescale $\tau_{\text {circ }}$ with the equation:

$$
-\frac{d \ln e}{d t}=\frac{1}{\tau_{\text {circ }}}
$$

(Zahn 1975). This equation implies that within a few $\tau_{\text {circ }}$ the binary motion assumes a nearly circular orbit, independently of its primordial eccentricity. We therefore expect any binary older than three or four times its $\tau_{\text {circ }}$ to become circular.

As shown by the seminal work of Zahn (1975), the circularization timescale is very sensitive to the orbital semi-major axis $a$ :

$$
\tau_{\text {circ }} \propto \begin{cases}\left(a / R_{1}\right)^{8} & \text { for stars with convective envelopes } \\ \left(a / R_{1}\right)^{13 / 2} & \text { for stars with radiative envelopes }\end{cases}
$$

where $R_{1}$ is the radius of the primary and $a$ is the binary semi-major axis, assuming the tidal dissipation in the secondary can be neglected. Determination of the eccentricities of short-period binaries, for which $\tau_{\text {circ }}$ is necessarily short, can therefore support the circularization idea observationally, if we find that all or at least most of the short-period binaries have circular orbits. Furthermore, considering a sample of coeval binaries, the eccentricity as a function of the binary period can tell us how the relevant $\tau_{c i r c}$ depends on the binary separation and the stellar structure.

\subsection{The SB9 sample of spectroscopic binaries}

One indirect evidence for long-term circularization processes can be obtained by considering the eccentricities of all known spectroscopic binaries as a function of their orbital period. To do that we use the 2751 systems compiled by the official IAU catalog of spectroscopic binaries - SB9 (Pourbaix et al. 2005), as of August 2007. The sample of the catalog is extremely inhomogeneous and includes stars of different spectral types and ages. In addition, the binaries compiled were discovered by different surveys with different observational instruments, rendering their discovery thresholds different as well. Furthermore, the compilation of the catalog 
is in progress, and therefore the catalog is not yet complete. Nevertheless, the catalog presents the largest sample of known spectroscopic binaries, and consequently we use it as a valuable source to study some features of the population of binaries in general, and short-period ones in particular.

Figure 1 shows the eccentricity of the SB9 binaries as a function of their period. Koch \& Hrivnak (1981) already plotted a similar plot, using a much smaller sample that included only late-type spectroscopic and eclipsing binaries known at that time. Similarly to the results of Koch \& Hrivnak, Figure 1 clearly shows that all binaries in the catalog with periods shorter than 0.35 days, with the exception of one binary, have circular orbits. In addition, all the binaries with longer periods except two, have their eccentricities below an 'upper envelope' that starts at eccentricity zero and climbs up to an eccentricity of 0.98 when moving from 0.35 to about 350 days. Just below the upper envelope the space diagram is somewhat sparse, and only below another stripe does the diagram density become high.

Somewhat arbitrarily, we chose to present the upper envelope and the sparse stripe by a function of the form

$$
f=E-A \exp \left(-(p B)^{c}\right) .
$$

The values of the parameters of the upper function are $E=0.98, A=3.25$, $B=6.3$ and $C=0.23$, and those of the lower function are $A=3.5$ and $B=3$.

One could argue that the paucity of eccentric short-period binaries above the upper envelope is the outcome of binary formation processes. However, the natural explanation for these two features - the circularized binaries and the existence of the envelope - is that they are the result of tidal circularization, which is substantially weaker in long-period binaries. This approach assumes that binaries were formed above the upper envelope in the diagram, but the circularization processes pushed them down.

If this is indeed the case, we still have to explain the existence of three systems high above the upper envelope: the cataclysmic variable WZ Sge, with a period of 0.057 days and an eccentricity of 0.17 (Walker \& Bell 1980), the binary pulsar PSR $1913+16$, with a period of 0.32 days and an eccentricity of 0.62 (Hulse \& Taylor 1975) and HD 142315 (Levato et al. 1987), a binary with a B8V primary, a period of 1.264 days and an eccentricity of $0.61 \pm 0.2$.

In fact, these three cases might not be a problem for the theory of circularization:

- The eccentricity of WZ Sge might not be real. The derivation of the radial velocities of WZ Sge was difficult, as the spectra were taken during the 1978 outburst, and the observed lines probably came from the outburst material around the white dwarf. In addition, the velocities derived from the He I lines are consistent with zero eccentricity (Walker \& Bell 1980).

- The binary pulsar is composed of two neutron stars with radii as small as $1 \mathrm{~km}$ (Hulse \& Taylor 1975). Therefore the circularization processes in that system are not in action at all. 


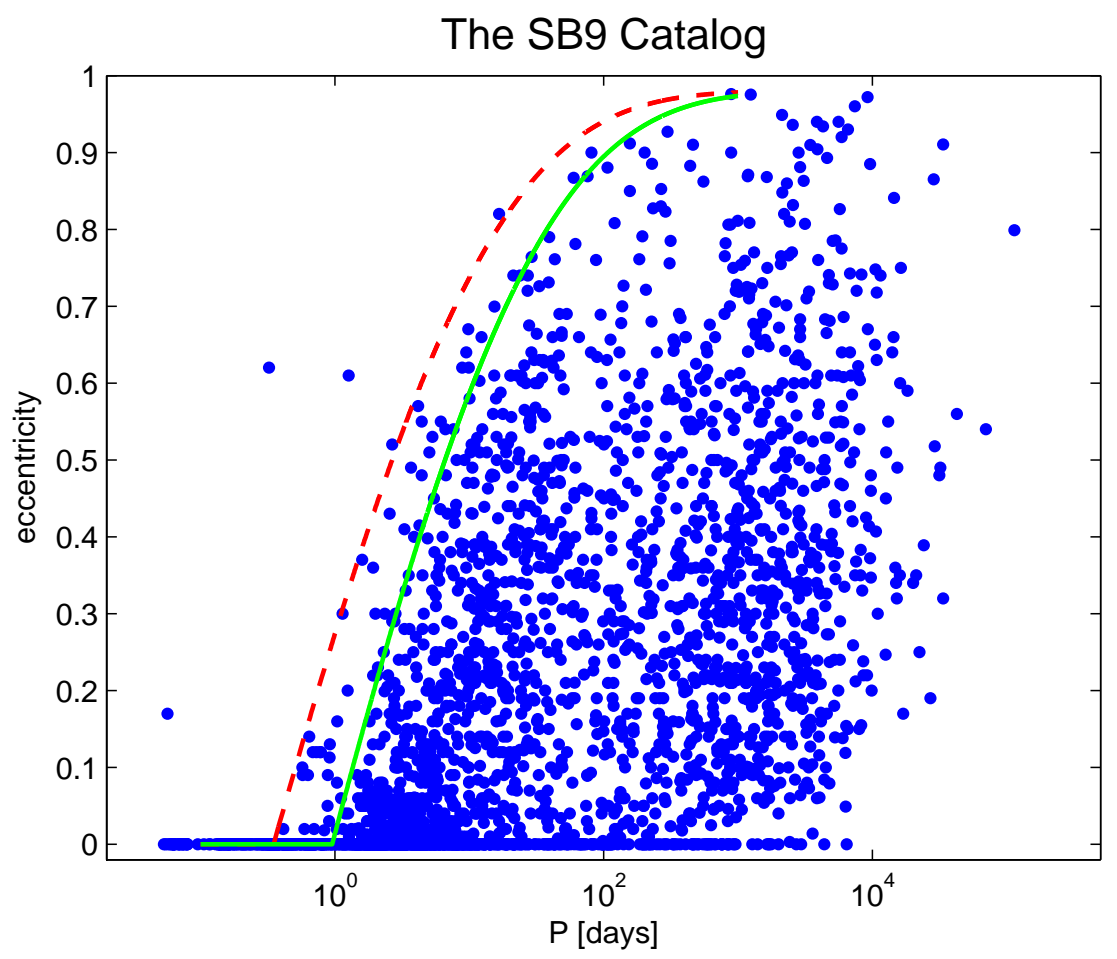

Fig. 1. The eccentricity of the spectroscopic binaries as a function of the orbital period. All 2751 binaries of the SB9 catalog are included. The red dashed line is the upper envelope, with an equation $f=E-A \exp \left(-(p B)^{c}\right)$, where $E=0.98, A=3.25, B=6.3$ and $C=0.23$. The green line is the line under which one can find most binaries, with $A=3.5$ and $B=3$

- The eccentricity of HD142315 is in fact $0.62 \pm 0.20$ (Levato et al. 1987), so the eccentricity is above the upper envelope by only one sigma.

Figure 1 can not be used for a quantitative study of the circularization processes, especially because of the lack of homogeneity and the incompleteness of the SB9 catalog. For such a study we will consider more homogeneous and coeval samples of stars below. Before diving into the quantitative consideration, we present in the next subsection two systems that show almost without any doubt an evidence for long-term circularization. We can acquire such an evidence only for circular binaries that we have strong reason to believe had eccentric orbits in the past. 


\subsection{X-ray binaries}

One of the first observational evidences for the long-term circularization processes taken place in short-period binaries was the discovery of the circular orbits of the first two close X-ray binaries - Cen X-3 (Schreier et al. 1972) and Her X-1 (Tananbaum et al. 1972), detected by Uhuru, the first X-ray satellite. These two X-ray pulsars were found in binaries with periods of 1.7 and 2.09 days, respectively, and with extremely small eccentricities. Later analysis of the data of Cen X-3 (Fabbiano \& Schreier 1977) indicated that its eccentricity is $0.0008 \pm 0.0001$ (see also a review by Bildsten et al. 1997). Such precise measurement of the eccentricity was possible only because of the rapid X-ray pulsation detected by the satellite, which enabled the observers to follow the orbit of the X-ray pulsar in great detail. The $\mathrm{X}$-ray pulsations come from a rapidly rotating neutron star (Davidson \& Ostriker 1973), formed by a supernova explosion that very probably produced substantial changes in the binary orbit, inducing a large orbital eccentricity (Wheeler et al. 1974; Chevalier 1975). Therefore, the observed minute eccentricity had to be associated with tidal circularization that was in action since the supernova explosion (Lecar et al. 1976).

In fact, the short periods of Cen X-3 and Her X-1 might themselves be another indication for tidal evolution taken place in these systems during their postsupernova phase. The reason for this is that the commonly accepted paradigm assumes that the neutron star in these systems was formed by a supernova type II, which occurred in the last stages of evolution of the original primary star, when it reached the supergiant phase (e.g., McCluskey \& Kondo 1971). The present binary system, with a separation of order of $0.1 \mathrm{AU}$, certainly could not accommodate a supergiant. Therefore, the binary orbits of Cen X-3 and Her X-1 have probably shrunk because of tidal evolution of the system (e.g., Sutantyo 1976), which led to transfer of angular momentum from the orbital motion to the present main-sequence star. Similar ideas were proposed for other X-ray binaries (e.g., Verbunt 1994; van Kerkwijk et al. 2000; Janssen \& van Kerkwijk 2005).

\subsection{Evidence for circularization in early-type binaries}

The circularization occurring in Cen X-3 brings evidence for tidal interaction in a binary with an early-type star, as the optical counterpart, V779 Cen (Krzeminski 1974), is of O6-7 II-III type (Ash et al. 1999), with a mass of about $20 M_{\odot}$. This is particularly important, as such an early-type star has a radiative envelope, whereas most of the stars in the SB9 catalog are cool stars with convective envelopes. Tidal dissipation is expected to be very efficient in convective envelopes, where the viscosity is high due to turbulent eddies (Zahn 1975). On the other hand, in early-type stars with radiative envelopes the dissipative processes are assumed to be radiative damping on the dynamical tide (Zahn 1977). Therefore, the circular orbit of Cen X-3 is an evidence for tidal interaction of a different kind from that which occurs in most of the binaries in the SB9 catalog.

The number of spectroscopic binaries with early-type stars is small because 
early-type stars have few spectral lines in their spectra, which makes it difficult to derive their radial velocities and discover their binarity. Additionally, very hot stars are rare in the solar neighbourhood, and therefore not many early-type stars are known as spectroscopic binaries.

In order to study the circularization processes in a larger sample of early-type stars, a seminal work of Giuricin et al. (1984) considered about 200 spectroscopic and eclipsing binaries with known $\mathrm{O}, \mathrm{B}$ and A primaries. They constructed their sample from the seventh catalog of spectroscopic binaries (Batten et al. 1978) and from the list of eclipsing binaries of Cester et al. (1979) and Wood et al. (1980). They plotted the eccentricity as a function of the orbital period, and showed that the two features of Koch \& Hrivnak (1981) do appear, although with different parameters. Excepting only a few cases, all binaries with periods shorter than 2 days are circular, and there is an 'upper envelope' that goes up to an eccentricity of 0.6 .

Giuricin et al. (1984) used the fact that the lightcurves of eclipsing binaries yield one additional parameter - the fractional radius, which is not available for spectroscopic binaries. This parameter gives the stellar radius as a fraction of the semi-major axis of the binary orbit. In fact, in early-type stars the circularization timescale strongly depends on the fractional radius (see Equation 4.3, taken from Zahn 1975). Therefore, Giuricin et al. (1984) plotted the eccentricity as a function of fractional radius for their sample, using a less accurate spectral estimation for the spectroscopic binaries. They have discovered that all binaries with fractional radius larger than 0.3 are circular, and there is an eccentricity 'upper envelope' that rises from zero to 0.8 when reducing the fractional radius from 0.3 to 0.05 . Similar results were found in modern photometric data of early-type binaries in the LMC, as discussed in Section 9.2 (see Figure 9 for a similar diagram).

The most natural interpretation of these findings is that systems with fractional primary radius larger than 0.3 have been circularized during the lifetime of the systems. Note, however, that Giuricin et al. found a few binaries with primary radius larger than 0.3 that displayed small but significant eccentricities. They attributed these eccentricities to unseen third distant companions, an effect suggested by Mazeh \& Shaham (1979; see detailed discussion in Section 7.2).

\subsection{Circularization in coeval sample of binaries}

The first evidence for circularization of coeval binaries was pointed out by the seminal work of Mayor \& Mermilliod (1984, hereafter MM84). They have plotted the eccentricities versus period of 33 solar-type main-sequence spectroscopic bina-

ries known at that time in a few open clusters, including the Hyades, Pleiades, Praesepe and Coma. MM84 found that the eccentricities of these binaries display a distinct change at a period of 5.7 days. All seven binaries with periods shorter than this critical period have circular orbits, while binaries with periods longer than 5.7 days have orbits with significant eccentricities.

The transition period between circular and eccentric binaries can be directly observed for a given sample of coeval binaries, though with considerable obser- 
vational effort. In principle, one can follow the radial velocities of a large coeval sample of similar stars, identify the binaries, determine their orbital parameters, the period and eccentricity in particular, and find the transition period. Since the seminal work of MM84, a few radial-velocity surveys have detected the transitionperiod effect. This was done for binaries with G-type primaries found in the solar neighbourhood (Duquennoy \& Mayor 1991), in the open clusters M67 (Latham et al. 1992a,b), NGC 188 (Mathieu et al. 2004) and recently M35 (Meibom \& Mathieu 2005, hereafter MM05), in PMS (Melo et al. 2001) and in the halo and field stars (Latham et al. 2002). These studies derived the transition periods for samples with different ages, starting with the PMS binaries, with an age close to zero, and moving up to the age of the Galactic halo, which is probably older than 10 Gyrs. The expectation was that transition periods lengthen in older samples, for which the circularization processes have been in action for a longer period of time. Therefore, the variation of the transition period as a function of the sample age can be used as indirect evidence of the circularization processes.

Following this line of reasoning, Mathieu \& Mazeh (1988, hereafter MM88) even suggested that the cutoff period can be used to estimate the sample age. However, it turned out that this idea can not be easily applied, as the theoreticians could not agree upon the details of the theory of circularization, as discussed below.

\subsubsection{The transition period}

Since the early days of circularization studies, the definition of the observed transition period between the circular and the eccentric orbits has been unclear. Is the transition period the longest period for which a circular binary was found, or is it the shortest period with an eccentric orbit? For example, Figure 2 shows the eccentricities of 18 binaries with short periods that were found by the seminal work of MM05 in the open cluster M35, out of their sample of 32 binaries. We somewhat arbitrarily define as circular binary any orbit with a derived eccentricity smaller than 0.05. This is indicated by the horizontal dotted line in the figure. According to this definition, the longest period with circular orbit is binary no. 4037, the one with the period of 16.5 days. On the other hand, the shortest period with an eccentric orbit is that of binary no. 0422, with 8.2 days (MM05). The two periods, delineated in the figure with vertical dashed lines, bound the possible range of the transition period. Such ambiguities exist for most observed samples.

In fact, the width of the transition range is not surprising. Mathieu and Mazeh (1988) already listed several factors that can blur the transition period between circular and eccentric binaries, even for a sample of binaries in which all systems have exactly the same primary mass. A major factor is the secondary mass, which might be different for the various binaries in the sample. After all, the gravitational attraction of the secondary is the source of the tidal force exerted on the primary, and therefore the tidal circularization timescale of the binary strongly depends on the binary mass ratio $q=M_{2} / M_{1}$, where $M_{1}$ is the primary mass and $M_{2}$ is the secondary mass. For a given period and primary star, Mathieu \& Mazeh (1988) got: 


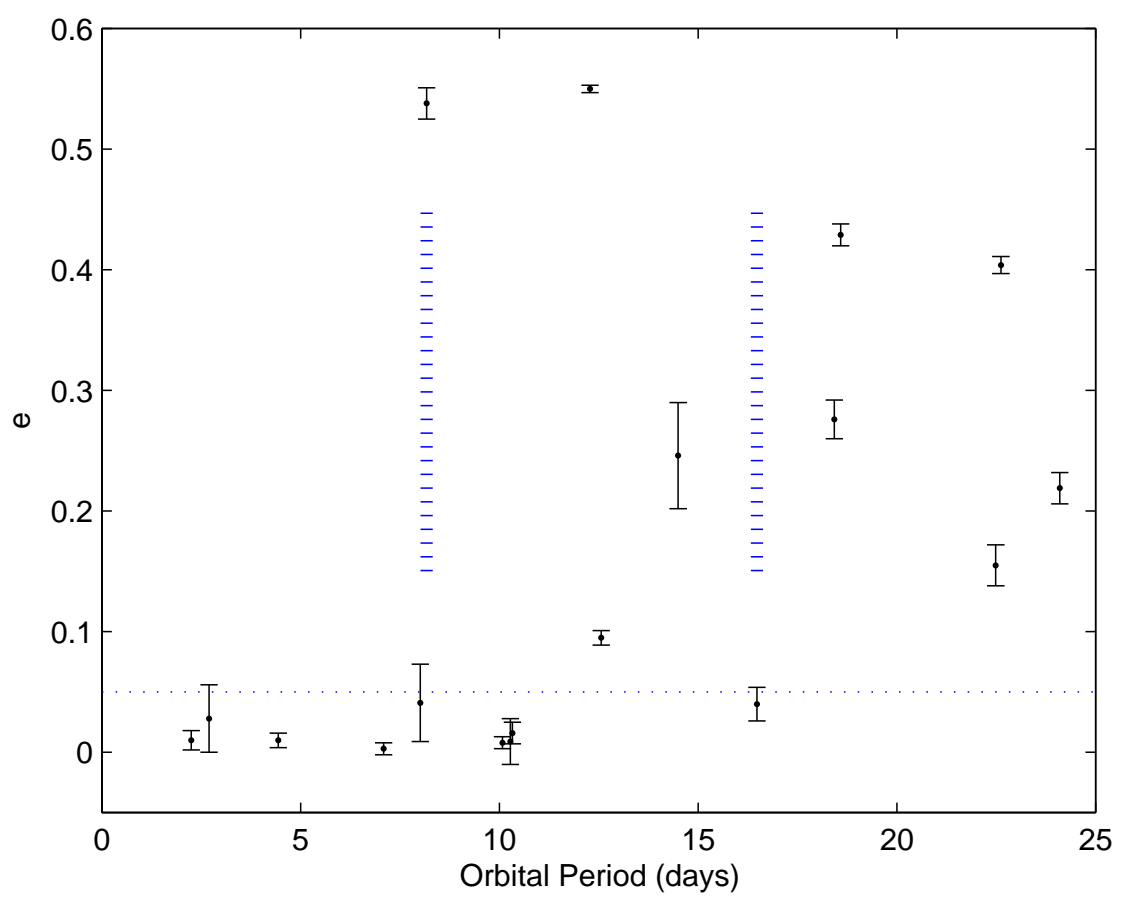

Fig. 2. The transition range between circular and eccentric binaries in M35, after MM05. All binaries with eccentricities below the horizontal dotted line are considered circular. The transition range is between the shortest period with an eccentric orbit and the longest period of circular binary

$$
\tau_{\text {circ }} \propto q^{2 / 3}\left(1+\frac{1}{q}\right)^{5 / 3}
$$

Therefore, the secondary mass can lengthen the timescale of the circularization processes taken place inside the primary by a factor of 4 , when moving from $q=1$ to $q=0.1$. Another factor to take into account is the circularization processes taking place in the secondary itself. For equal masses, the secondary contributes to the circularization processes as much as the primary. But when we move to a binary with a mass ratio of, say, 0.5 , the circularization processes in the secondary are negligible, because of the small stellar radius. Considering the two effects together, the secondary mass can change the binary circularization timescale by a factor of 8 , which corresponds to a factor of 1.5 in circularization period, if we assume that $\tau_{\text {circ }} \propto P^{16 / 3}$ (Zahn 1977).

As radial-velocity observations typically are not capable of determining the nature of the secondary star in single-lined spectroscopic binaries, the spread of 
mass ratios in any sample of spectroscopic binaries induces a spread of the typical circularization timescales.

Another factor that can change the time needed for a binary to reach circularization is its primordial eccentricity. For example, a binary with initial eccentricity of 0.75 needs twice the amount of time needed by a binary with initial eccentricity of 0.2 to get to an eccentricity of 0.05 . Therefore, a single circular binary with a long period could have been the result of a low initial eccentricity, and not necessarily because of the efficiency of the circularization processes.

In addition, samples of spectroscopic binaries are not uniform, and the primary masses are not always the same. A small change in the primary mass can dramatically change its interaction with the varying tidal pull of its secondary.

Lastly, triple systems can show the Mazeh \& Shaham (1979) effect, by which a third distant star can pump eccentricity into the binary orbit (see Section 7 below), even if the binary system starts with a circular orbit. Mayor \& Mazeh (1987) have shown that many of the spectroscopic binaries might have third faint companions (see a more detailed discussion in Section 7). Furthermore, Tokovinin et al. (2006; see also Tokovinin 2004) claimed that more than $90 \%$ of the shortperiod binaries, with periods shorter than 3 days, have at least one additional companion. Therefore, the Mazeh \& Shaham effect can be quite frequent (see Section 7.2 for a detailed account of the observational evidence for this effect), causing (small) eccentricities to appear in short-period binaries.

Because of all these considerations, the blur of the transition between circular and eccentric periods in any sample is inevitable. Therefore, a transit range between the circular and the eccentric binary is not that surprising. Nevertheless, some researchers used the term 'cutoff period' to denote the longest period of a circular orbit in the sample; see, for example, Melo et al. (2001) who suggested that the transition period of PMS stars is 7.56 days, based on one circular binary. Such an approach should be modified, given the different factors that might blur the transition.

\subsubsection{Derivation of the transition period - Meibom \& Mathieu approach}

A major step in the discussion of the transition period was recently achieved by Meibom and Mathieu (2005), who came up with a new algorithm to derive a transition period for a sample of spectroscopic binaries. Instead of expecting the sample to be drastically divided between circularized and eccentric binaries, MM05 defined a new expected 'averaged' eccentricity, which presents a smooth transition between the two parts of the sample. Based on extensive simulations of populations of binaries, they concluded that the final 'averaged' eccentricity as a function of their period is of the shape

$$
e(P)=\left\{\begin{array}{cc}
0 & \text { if } P \leq P_{\text {tran }} \\
\alpha\left(1-e^{\beta\left(P_{\text {tran }}-P\right)}\right)^{\gamma} & \text { if } P>P_{\text {tran }} .
\end{array}\right.
$$

In this distribution the eccentricity assumed zero value up to a 'transition' period, $P_{\text {tran }}$, and then climbed exponentially to the averaged eccentricity of $\alpha$. The 
Table 1. Circularization Periods for different coeval samples derived by MM05

\begin{tabular}{lcc} 
Binary Population & $\begin{array}{c}\log \text { Age } \\
\text { (Gyrs) }\end{array}$ & $\begin{array}{c}\text { Transition Period } \\
\text { (days) }\end{array}$ \\
\hline PMS & -2.5 & $7.1 \pm 1.2$ \\
Pleiades & -1.0 & $7.2 \pm 1.8$ \\
M35 & -0.8 & $10.2 \pm 1.2$ \\
Hyades/Praesepe & -0.2 & $3.2 \pm 1.2$ \\
M67 & 0.6 & $12.1 \pm 1.2$ \\
NGC188 & 0.8 & $14.5 \pm 1.8$ \\
Nearby G primaries & 0.95 & $10.3 \pm 2.3$ \\
Halo & 1.00 & $15.6 \pm 2.8$ \\
\hline
\end{tabular}

parameters $\beta$ and $\gamma$ controlled the steepness of the rise of the averaged eccentricity. Based on their simulation they adopted the values of $\beta$ and $\gamma$ to be 0.14 and 1.0, respectively. MM05 found that the mean value of the eccentricity of the longperiod binaries in all samples they considered was 0.35 , and therefore they adopted this value for $\alpha$.

To derive the transition period of a sample, MM05 suggested finding the $P_{\text {tran }}$ parameter of their function that best fits the sample periods and eccentricities. The advantage of their approach was that $P_{\text {tran }}$ did not depend on one binary alone, with the longest circular period or the shortest eccentric period, but, instead took the whole set of eccentricities and periods in the transition range into consideration.

In the course of their thorough study, MM05 analysed eight available samples of coeval spectroscopic binaries with their approach, and derived transition periods for each of these samples. Their results are given in Table 1. where we round the uncertainties of the periods.

Each of the eight samples has similar binaries and is comprised of mainsequence G-type primaries. It should therefore be interesting to compare the derived transition period of the samples as a function of their age. We followed MM05 and plotted their result in Figure 3. Based on the discussion above with regard to the factors that can blur the transition between the circular and eccentric binaries, we did not use MM05 errors. Instead, we assign somewhat arbitrarily to each transition period an error of $50 \%$ up and $33 \%$ down, so the up and down errors are the same in logarithmic scale.

Two features are emerging from the thorough analysis of MM05, as presented in Table 1 and Figure 3. First, for seven out of the eight coeval samples discussed by MM05, the transition period is between 6 and 16 days. Second, for the same seven samples, the transition period seems to be a monotonic increasing function of the sample age. The only outlier which is not consistent with these two features is the sample composed of the binaries found in the Hyades and Praesepe, two clusters of the age of 650 Myrs. The MM05 analysis of this sample yields a transition 


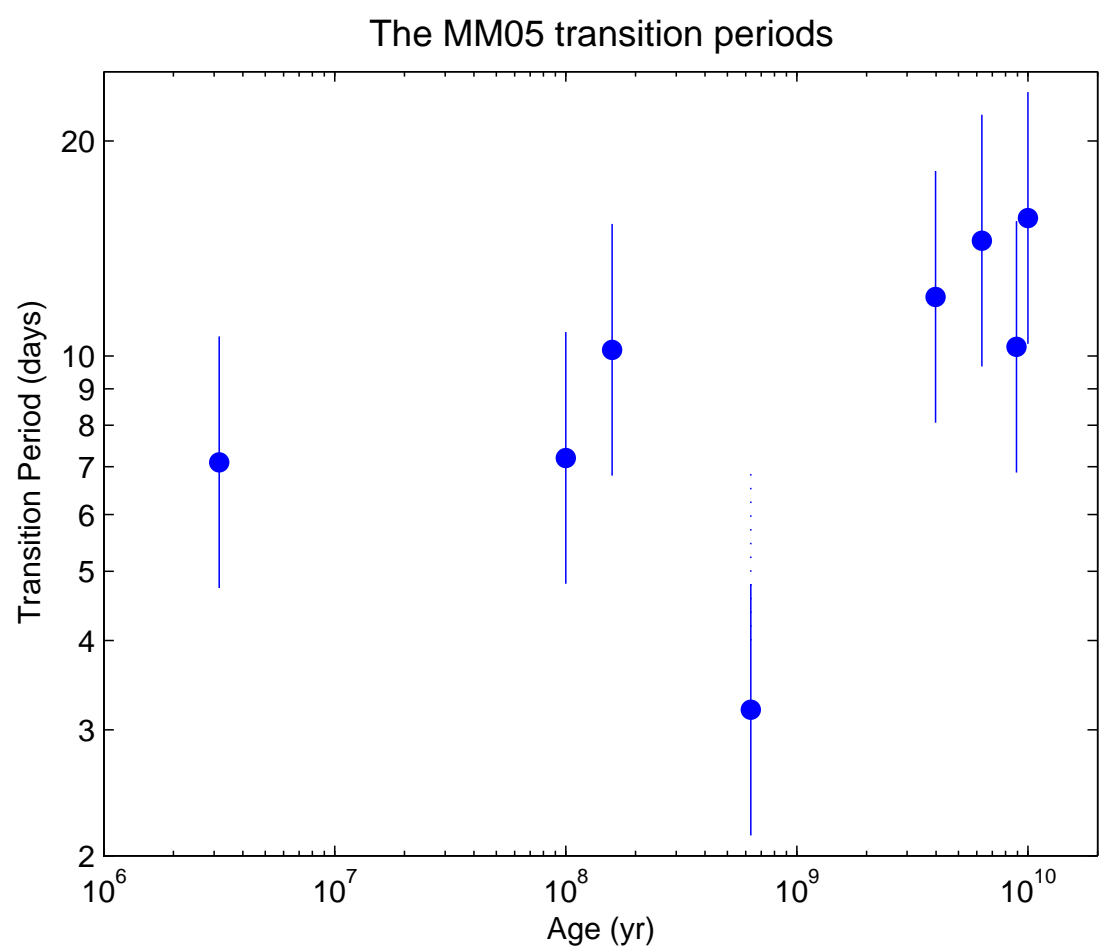

Fig. 3. The transition period for 8 coeval systems as a function of their age, after MM05. Contrary to MM05, we assigned to each period an error of factor of $50 \%$ (see text). The error bar of the transition period of the Hyades/praesepe sample was extended by a dotted line (see text).

period of 3.2 days, but this transition period is strongly affected by two binaries. One binary is KW 181 (Mermilliod \& Mayor 1999), with a period of 5.9 days and an eccentricity of 0.36 , and the other is $\mathrm{vB} 121$, with a period of 5.75 days and an eccentricity of 0.35 (Griffin \& Gunn 1978). Without these two systems, the algorithm of MM05 would assign a transition period of about 7 days to the Hyades and Praesepe sample. To mark this fact, we lengthened arbitrarily the error bar of the symbol that presents the Hyades and Praesepe sample in the figure. We therefore conclude that the accumulated observational data are consistent with the assumption that most, if not all, transition periods of coeval samples are longer than 6 days, and there seems to be a tendency for longer transition periods in older clusters. 


\subsection{Confrontation of the theory with the observations}

The analysis of MM05 makes it possible to compare the derived transition periods with the theory. Three theoretical models were suggested for the tidal dissipation acting in close binary systems:

- The theory of equilibrium tide, which assumes that the stellar tidal bulge, induced by its companion, lags at some angle after the orbital motion of the companion. The lag, which is due to dissipative processes inside the star, enables a transfer of energy and angular momentum from the orbital motion to the stellar rotation (Zahn 1966). This mechanism is mostly effective in stars with convective envelopes, and therefore the equilibrium tide was applied only to binaries with late-type stars (Zahn 1977).

- The theory of dynamical tides, which assumes that the tidal interaction is acting through the damping of gravity modes inside the stellar radiative zone (Zahn 1970; 1975; Savonije \& Papaloizou 1983, 1984). It was assumed that this mechanism is only effective in stellar radiative zones, and therefore was applied first to high-mass stars with radiative envelopes exclusively (Zahn 1977). However, in the last decade a few studies (e.g., Terquem et al. 1998; Goodman \& Dickson 1998; Witte \& Savonije 2002; Savonije \& Witte 2002) have shown that this mechanism can be effective in radiative cores of latetype stars as well. Very recently tidal interaction with inertial waves in convective envelopes of solar-type stars was also considered as a source for orbital evolution (Ogilvie \& Lin 2007; see also Ivanov \& Papaloizou 2007).

- The theory of hydrodynamic flows, which assumes that the tidal forces of the companion induce large-scale meridional flows of the stellar surface, which in turn exert torques on the companion (Tassoul 1995). This theory was questioned by Rieutord \& Zahn (1997) and was later defended by Tassoul \& Tassoul (1997).

Each of these theories should account for the overall result presented above which gives an absolute scaling of the tidal circularization processes in a binary with G-type primary. The observationally derived transition periods for the different coeval samples imply that for a binary with a period of about 10 days the circularization timescale is of the order of $10^{9} \mathrm{yrs}(=\mathrm{Gyr})$. The theories should be able to account for the possible mild increase of the transition period as a function of sample age, presented already in MM88, and discussed by MM05 in particular.

The plot of MM05 is of great interest because the slope of the period increase can, in principle, distinguish between the different theories. This is particularly true if we consider several updated versions of the equilibrium tide theory. This theory was restudied by Zahn (1989), who specifically considered the reduction of the turbulent viscosity when the tidal period becomes shorter than the convective turnover time. He concluded that the circularization timescale should be $\tau_{\text {circ }} \propto P^{16 / 3}$. In a follow-up paper, Zahn and Bouchet (1989) concluded that all the circularization of G-type binaries must occur during the pre-main-sequence 
(=PMS) phase, when the stars are substantially larger than they will be during their main-sequence phase. They further claimed that the circularization during the main-sequence phase is negligible, and they therefore predicted that the circularization period would be between 7.2 and 8.5 days for all samples, regardless of their respective ages.

Not all studies agreed with Zahn (1989) approach to the reduction of the turbulent viscosity. Goldman \& Mazeh (1991), for example, proposed a modified approach that resulted in $\tau_{\text {circ }} \propto P^{10 / 3}$. They also pointed out the evidence that was available at that time for the variation of the transition period with the sample age. Goldreich \& Nicholson (1989) and Goodman \& Oh (1997) studies yielded power dependence which is in between that of Goldman \& Mazeh and that of Zahn (1989).

It seems that the plot of MM05 (Figure 3) indicates that the circularization processes during the PMS phase do circularize binaries up to about 6-8 days, as Zahn and Bouchet suggested. It also suggests that transition periods probably become longer in older samples, in contrast to the other claim of Zahn \& Bouchet (Goldman \& Mazeh 1991; Mathieu et al. 1992), although the slope of the figure is not conclusive enough to distinguish between the different theories. If this is true, we must find out why the theory of equilibrium tide, when applied to main-sequence stars, yields a circularization absolute efficiency which is too low to explain the observations (e.g., Goodman \& Oh 1997; Terquem et al. 1998; Goldman \& Mazeh 1991; Sasselov 2003). We therefore have to close this discussion with the banal statement that we need more data for a definite conclusion.

\subsection{Circularization of binaries with giant star component}

An additional line of evidence for tidal circularization was studied by Verbunt \& Phinney (1995, hereafter VP95), who considered the eccentricity as a function of orbital period of binaries containing a giant component found in open clusters. VP95 noted that giants can serve as the simplest test cases for the circularization theory, because the orbital period of such systems is much larger than the eddy timescale, and therefore we do not need to worry about the reduction efficiency of the viscosity. Furthermore, giants are fully convective and therefore avoid the problem of tides in the radiative zone. We note that the large radii of the giants render the circularization during PMS phase irrelevant, another advantage of studying binaries with giants. It is true that the giants have an history of fast expansion, which causes the circularization timescale to change rapidly. However, VP95 claimed that from the cluster age and turnoff mass one can reconstruct the history of the giants and integrate the expected theoretical circularization effect.

The sample of VP95 included 28 binaries from 12 open clusters, taken from the works of Mermilliod \& Mayor $(1989 ; 1990)$, Mermilliod et al. (1989) and Mathieu et al. (1990). They found that except two systems, all binaries with periods shorter than 200 days have circular orbits. However, as the systems are with different ages and masses, the orbital period is not the best parameter to compare the observed eccentricity with. When VP95 considered instead the expected eccentricity reduc- 
Table 2. The five most eccentric spectroscopic binaries in SB9. The table includes the period and eccentricity of each system and also the 'periastron period', $P_{\text {peri-dist }}$ - the orbital period of a circular orbit with a radius that equals the actual periastron distance.

\begin{tabular}{lrccl} 
Binary & $e$ & $\begin{array}{c}\text { Orbital Period } \\
(\text { days })\end{array}$ & $\begin{array}{c}P_{\text {peri-dist }} \\
(\text { days })\end{array}$ & Reference \\
\hline HD 2909 & 0.949 & 2128 & 25 & Mazeh et al. 1995 \\
& \pm 0.002 & & & \\
HD 165590 & 0.958 & 7400 & 64 & Batten et al. 1979 \\
& \pm 0.001 & & & Udry et al. 1998 \\
HD 123949 & 0.972 & 9200 & 43 & \\
& \pm 0.057 & & 3.5 & Duquennoy et al. 1992 \\
Gliese 586A & 0.9752 & 890 & & Tokovinin et al. 2003 \\
& \pm 0.0003 & & 4.8 & \\
41 Dra & 0.9754 & 1250 & &
\end{tabular}

tion, based on the history of the giants derived from their mass and position on their H-R diagrams, they concluded that all are consistent with Zahn theory.

More than a decade has passed since the seminal work of VP95, and many more giants have been discovered since. A new study of the binaries with giants in open clusters and in the field is highly due, so we can learn more about the circularization processes and derive their absolute scaling of efficiency.

\subsection{Highly eccentric binaries and the theory of circularization}

Some of the main-sequence spectroscopic binaries were found to have very high eccentricities. The five most eccentric binaries in SB9, with an eccentricity larger or equal to 0.95 , are compiled in Table 2, which lists their orbital periods and eccentricities. The two most eccentric systems might be a challenge to the circularization theory, as their periastron distance is quite small, and therefore we would expect them to circularize, or at least to substantially reduce their eccentricities on a relatively short timescale (Duquennoy et al. 1992).

To estimate the effectiveness of the tidal forces at periastron, I introduce the 'periastron period', $P_{\text {peri-dist }}$ - the orbital period of a circular orbit that has the same orbital radius as the actual periastron distance. It is easy to show that

$$
P_{\text {peri-dist }}=P(1-e)^{3 / 2} \text {. }
$$

Note that this is not the period that presents the orbital angular velocity at periastron. Table 2 lists the corresponding periastron periods of the five systems.

¿From the table we see that two of the systems, with eccentricities larger than 0.97 , indeed have short periastron distances, which are equivalent to orbital periods shorter than 5 days. If these systems are of the age of a few Gyrs, we would naively 
expect them to circularize, or at least to reduce their eccentricities during their main-sequence lifetime.

Goldman \& Mazeh (1994), who studied the tidal history of Gliese 586A, argued that for highly eccentric binaries the tidal shear, which drives the turbulent viscosity, changes near periastron on a timescale shorter than the convective timescale. Therefore the turbulent viscosity (e.g., Hut 1982a;b) is substantially reduced, as was argued in Section 4.5. With their recipe for the reduction of turbulence, Goldman \& Mazeh were able to suggest a tidal history of Gliese 586A that leads to the present parameters of the system. Obviously, similar arguments can be applied to the other systems.

Goldman \& Mazeh even argued that the highly eccentric binaries have the potential for distinguishing between the different recipes for viscosity reduction. Unfortunately, very eccentric binaries are difficult to discover, as most of their radial-velocity variation is concentrated in a very small part of the orbital period (e.g., Griffin 1984). Therefore only very few highly eccentric binaries are known. Nevertheless, I suspect that many of these binaries exist, and when they are discovered we will be able to use them as test cases for tidal interaction.

\section{Synchronization}

As stated in the introduction, tidal forces in binaries also tend to synchronize the rotation of the two stars with the orbital motion. The theory of tidal evolution (Zahn \& Bouchet 1989; Witte \& Savonije 2002) predicts that the synchronization timescale will be two or three orders of magnitudes shorter than the circularization timescale. This has to do with the fact that the angular momentum associated with stellar rotation is much smaller than the orbital angular momentum. Consequently, the angular momentum transferred between the orbital motion and the stellar rotation in order to reach synchronization is much smaller than that which is needed to attain circularization. This means that we can expect binaries to reach synchronization a long time before they reach circularization. Similarly to circularization, we should also expect coeval sample of binaries to show a transition period between synchronized and non-synchronized binaries. Such an observed transition can serve as another confirmation of the theory of tidal interaction.

However, both the observations and the theory of stellar synchronization are more complicated than those of tidal circularization. Even the term 'stellar rotation' is not well defined, as the notion of a star rotating as a rigid body is obviously an oversimplification. For example, differential rotation as a function of stellar latitude (e.g., Lyytinen et al. 2002; Croll et al. 2006; Walker et al. 2007) must be taken into account when we define stellar rotation. Therefore the following section adopts an observational approach, according to which the stellar rotation is the observed rotation, and which disregards complications induced by differential rotation.

The stellar rotational period can be obtained either by photometric or spectroscopic observations. Photometric monitoring can reveal stellar periodic variability, which presumably is caused by stellar spots that come into and go from the facing 
hemisphere of the star, as the star rotates on its axis (e.g., Stauffer et al. 1987). Stellar spectra, with high enough resolution and S/N ratio, yield line broadening which can be modelled to yield stellar rotational velocity. With the knowledge of the size of the stellar radius and inclination angle it is possible to derive the stellar rotation period (e.g., Stauffer et al. 1984). However, as stellar radii and inclinations are usually not well known, the spectroscopic approach in most cases induces a large uncertainty of the rotational period. On the other hand, in spectroscopic binaries the spectra have already been obtained, in contrast to the photometry that usually necessitates additional observational effort.

The variability method is dependent upon the longevity of the spots. We assume that although stellar spots indeed form and dissolve, they still maintain some coherence with a timescale much longer than the stellar rotation period. If this is the case, then the spots can maintain a periodic brightness variability, modulated with the rotation period. However, as stellar spots form and dissolve, the amplitude and phase of the modulation might not be constant (see, for example, a discussion of the stellar spots of V1794 Cyg by Jetsu et al. 1999). This can cause some spread of the frequency power of the modulation over neighbouring frequencies, rendering the detection of the periodic modulation more difficult. Therefore many measurements are needed in order to detect the rotational frequency, preferably distributed over many rotational periods.

Another complication faced by the photometric approach is the existence of other sources of variability, coming either from the star itself, like stellar pulsation, or from the photometric modulation of its companion. The latter is specifically true for binaries with comparable brightness, where the secondary modulation can have an amplitude that is comparable with that of the primary. In addition, the ellipsoidal variability, with half the binary period, can also be present in the photometric data (see, for example, the analysis of the RS CVn-type stars RT And by Zhang \& Gu 2007 and SZ Psc by Eaton \& Henry 2007). Therefore, the identification of stellar rotation by a periodic modulation should be done with extra caution, and only after other sources of periodic modulation have been excluded.

On the theoretical side, whereas for the eccentricity evolution only the tidal interaction is in action, stellar rotation has its own evolution even in single stars. Contraction of PMS stars that are approaching the main-sequence phase and their interaction with their accretion disc (e.g., Königl 1991; Shu et al. 1994) can change the stellar rotation substantially (for observational evidence see, for example, Choi \& Herbst 1996 and Stassun et al. 1999). In addition, the rotational angular momentum itself can evolve during the main-sequence phase (e.g., Baliunas et al. 1995), via magnetic coupling with the stellar wind, for example.

Because of the difficulties of the theory and observation, less attention has been paid by the binary community to the study of tidal synchronization. Two exceptions are the works of Abt et al. (2002) and Abt \& Boonyarak (2004), who studied samples of B and A stars known to reside in binaries. Abt et al. (2002) derived the rotational period of their samples from the stellar line broadening, and concluded that all B-type stars with orbital periods shorter than 2.4 days are synchronized, while binaries with periods between 2.4 days and 5.0 days are 
'nearly synchronized'. The corresponding period ranges for A-type stars are 4.9 and 10.5 days, or twice as large. They also found that the rotations of the primaries are synchronized earlier than their orbits are circularized. The maximum orbital period for circularized $\mathrm{B}$ binaries is 1.5 days and for A binaries is 2.5 days. Abt et al. suggested that their finding is consistent with the theory of Zahn for early-type stars.

Abt \& Boonyarak found that B and A stars in binaries with periods as long as 500 days have rotational period significantly shorter than the corresponding single stars. They therefore concluded that the synchronization processes can have impact on the stellar rotation even for relatively wide binaries with periods as long as 500 days. The origin of these findings is still not clear, and needs further theoretical study.

The results of these two studies are most interesting, despite the difficulties to derive the rotational stellar periods, specially because these two studies focus on early-type stars (see Subsection 4.3 for discussion of the very few studies of circularization of early-type stars). We wish that similar studies would have been performed for $\mathrm{G}$ and $\mathrm{K}$ stars, and the results confronted with the theory. We could benefit from enlarging the interaction between theory and observations to include both circularization and synchronization of both early- and late-type stars.

Note that because the synchronization timescale is probably substantially shorter than that of circularization, the synchronized binaries might be more common than the circularized orbits (see above). This means that we can find many synchronized binaries with eccentric orbits. However, for eccentric binaries the orbital angular velocity is not constant, and therefore the synchronization frequency is not well defined. There is no rotating frame of reference attached to the primary for which the secondary is at rest. Therefore, eccentric binaries need a new definition of synchronization which will be reviewed in the next subsection.

\subsection{Pseudo-synchronization}

As the tidal forces strongly depend on the distance between the two stars, it is clear that in eccentric binaries each star should respond mostly to the orbital angular velocity near the periastron, where its companion is the closest. In a seminal paper, Hut (1981) has demonstrated that in the so-called weak friction approximation (see Zahn in this volume), each star achieves its equilibrium when it rotates with a frequency that is smaller but still close to the orbital periastron frequency. The equilibrium frequency, which Hut calls the pseudo-synchronization frequency, is

$$
n_{\text {pseudo }}=\frac{1+\frac{15}{2} e^{2}+\frac{45}{8} e^{4}+\frac{5}{16} e^{6}}{\left(1+3 e^{2}+\frac{3}{8} e^{4}\right)\left(1-e^{2}\right)^{3 / 2}} n_{\text {orbit }},
$$

where $n_{\text {orbit }}=2 \pi / P$ is the averaged orbital frequency. Sometimes it is convenient to express the pseudo-synchronization frequency with the angular velocity at periastron $-n_{\text {peri }}$ :

$$
n_{\text {peri }}=\frac{(1+e)^{1 / 2}}{(1-e)^{3 / 2}} n_{\text {orbit }}
$$


Table 3. The PMS and M35 young cluster eccentric systems with their rotation periods from Marilli et al. (in the first part of the table) and Meibom et al. (below the horizontal line in the table). For each system the pseudo-synchronized period is calculated according to the Hut (1981) recipe. The last system is the only circular system with a non-synchronized rotational period from M35.

\begin{tabular}{lcccc} 
Binary & $\begin{array}{c}\text { Orbital } \\
\text { Period } \\
\text { (days) }\end{array}$ & e & $\begin{array}{c}\text { Pseudo-synch. } \\
\text { Period } \\
\text { (days) }\end{array}$ & $\begin{array}{c}\text { Rotational } \\
\text { Period } \\
\text { (days) }\end{array}$ \\
\hline $044059.2-084005$ & 13.56 & 0.22 & 10.5 & 2.75 \\
$053043.1-043453$ & 40.57 & 0.32 & 24.7 & 12.90 \\
$053202.1-073153$ & 46.85 & 0.47 & 18.4 & 3.44 \\
$0539.8-0205$ & 18.74 & 0.39 & 9.4 & 4.48 \\
\hline $06091557+2410422$ & 8.17 & 0.65 & 1.6 & 3.71 \\
$06095563+2417454$ & 30.13 & 0.27 & 20.8 & 2.84 \\
$06085441+2403081$ & 12.28 & 0.55 & 3.7 & 6.03 \\
$06090257+2420447$ & 10.28 & 0.001 & 10.3 & 2.3
\end{tabular}

which leads to the expression

$$
n_{\text {pseudo }}=\frac{1+\frac{15}{2} e^{2}+\frac{45}{8} e^{4}+\frac{5}{16} e^{6}}{\left(1+3 e^{2}+\frac{3}{8} e^{4}\right)(1+e)^{2}} n_{\text {peri }}
$$

(Hut 1981). To third order of the eccentricity $e$ one gets:

$$
n_{\text {pseudo }} \simeq\left(1-2 e+7.5 e^{2}-13 e^{3}\right) n_{\text {peri }}=\left(1+6 e^{2}\right) n_{\text {orbit }} .
$$

Only subsequent to the work of Hut (1981) can we examine the eccentric orbit for its pseudo-synchronization. Of particular interest are coeval samples of binaries with a transition between the pseudo-synchronized and non-pseudo-synchronized binaries.

\subsection{Synchronization of young stars}

Two important studies of synchronization of short-period binaries have been performed recently. Marilli et al. (2007) photometrically observed 40 PMS T Tauri stars that were discovered as X-ray sources by the ROSAT Survey of the Orion complex, and subsequently were observed optically with both low and high resolution spectroscopy. They obtained extensive photometric data and analysed them with the Lomb-Scargle periodogram (Lomb 1976; Scargle 1982), deriving photometric modulation periods for 39 stars. Assuming the modulations are due to spots, these periods present the stellar rotational periods. Meibom et al. (2006) derived the rotation periods of 13 close binaries in M34 and M35, two young open clusters, with an age of 150 and 250 Myrs, respectively. 




Fig. 4. Rotational periods as a function of the orbital periods for eight PMS binaries (Marilli et al. 2007) and six young stars (Meibom et al. 2006). Circular binaries are encompassed by a circle. The dashed line is the locus of points for which the rotation period equals to the orbital period.

Eight of the stars observed by Marrilli et al. were found to be in spectroscopic binaries (Covino et al. 2001). Four systems, with orbital periods shorter than 10 days, were found to be circular and synchronized. The other four binaries, with orbital periods between 10 and 50 days, with eccentric orbits, are listed in the upper part of Table 3 . The table shows the orbital, rotational and pseudo-synchronization periods. It is easily observed that the rotational periods are substantially shorter than the pseudo-synchronization periods.

Six systems from the Meibom et al. (2006) study have periods shorter than 100 days, and of the six, three systems have eccentric orbits. These systems are included in Table 3, together with the system $06090257+2420447$, for which the orbit is circular but is very far from synchronization. All binaries in the two studies with periods shorter than 100 days, including those which are documented in the table and another six circular and synchronized binaries, are plotted in Figure 4.

Both samples are extremely small, but one feature seems to emerge from the figure: the transition period between circular and eccentric orbits is between 8 and 10 days. It would be reasonable to expect that the transition between pseudosynchronized and non-pseudo-synchronized binaries should appear on much longer period, as the synchronization processes are a few orders of magnitude more effi- 
cient. However, the striking feature of this figure is that the transition between pseudo-synchronized and non-pseudo-synchronized systems is at about the same period!

To understand this surprising similarity between the two transition periods we must bear in mind that while the circularization processes worked undisturbed during the PMS phase with high efficiency (Zahn \& Bouchet 1989), the synchronization processes were drastically affected by the internal variation inside the stars which changed their radii and therefore their rotational period. The first 100 Myrs of M35 after the stars settled on the main sequence were probably not enough to achieve pseudo-synchronization for binaries with periods longer than 810 days. In order to observe the strong potential of the synchronization processes, we apparently need to observe much older clusters.

Note that for the two most eccentric systems in Table 3, with eccentricity of 0.55 and 0.65 , the pseudo-synchronized period is shorter than the actual rotational period of the primary star. This is in contrast to the other five systems with more moderate eccentricities, for which the pseudo-synchronized period is longer than the rotational period. If this feature is validated by further observations, the difference between the highly eccentric binaries and the binaries with small or medium eccentricities could be a noteworthy feature (see, however, the discussion of V1143 Cyg in Section 5.3.1. The observed longer rotational periods could be associated with the approximation used by Hut in his work, which did not take into account the reduction of the turbulent viscosity on a timescale shorter than the convective timescale (Goldman \& Mazeh 1994). It is possible that the pseudosynchronization periods for some stars in highly eccentric orbits are longer than the ones derived by Hut's formula. It is therefore extremely important to derive stellar rotational periods for many more highly eccentric binaries and to compare them with the pseudo-synchronized periods.

\subsection{Interesting cases}

\subsubsection{V1143 Cyg}

V1143 Cyg is a bright, nearby, eclipsing double-lined spectroscopic binary, and therefore allows, in principle, for a determination of the masses, radii and luminosities of the two stars (e.g., Popper 1980). The binary consists of two F5V stars orbiting each other with a period of 7.6 days and an eccentricity of 0.54 (Snowden \& Koch 1969). Apparently, at the age of 2 Gyrs, the system did not have enough time to circularize (Andersen et al. 1987). Andersen et al. derived the rotational broadening of the two components to be $V_{A} \sin i_{A}=18 \pm 3$ and $V_{B} \sin i_{B}=28 \pm 3$ for the primary and secondary, respectively. With the derivation of the two radii $-1.346 \pm 0.023$ and $1.323 \pm 0.00023$ solar radii for the primary and secondary, and assuming an inclination close to $90^{\circ}$, one gets for the rotation period of the two stars 3.8 and 2.3 days, respectively. Andersen et al. results are confirmed by the very recent detailed study of Albrecht et al. (2007).

Andersen et al. (1987) pointed out that the pseudo-synchronization period, 
derived from the orbital period and the eccentricity, is 2.3 days, exactly equal to the rotation period of the secondary. Therefore, V1143 Cyg is a good example of a binary that has reached pseudo-synchronization, at least for one star, but obviously has not yet reached circularization. Thus, V1143 Cyg could be considered one of the few binaries that strongly supports Hut's (1981) theory of pseudo-synchronization.

However, the rotational period of the primary is a challenge to the theory. It is hard to conceive how the secondary could have been pseudo-synchronized while the primary was not, especially since the finding of Andersen et al. (1987) indicates that the primary and the secondary are extremely similar. Is it possible that the initial rotation of the primary and the secondary were so different that there was enough time to pseudo-synchronize the secondary but not the primary? I find such an explanation hard to believe. If it is indeed true, the disparity between the rotational periods of the two components remains a mystery. The primary of V1143 Cyg joins the group of highly eccentric binaries with rotational periods longer than Hut's pseudo-synchronization period, which was discussed above.

Recent work on the inclination of the two stars of V1143 Cyg relative to the orbital plane is reviewed in Section 6.2.2

\subsubsection{KH 15D}

$\mathrm{KH} 15 \mathrm{D}$ is a T Tau binary star in the young cluster NGC 2264 (Badalian \& Erastova 1970) that fades by three mag every 48 days (Kearns \& Herbst 1998). The best model to account for the many observed details of the periodicity of $\mathrm{KH}$ $15 \mathrm{D}$ assumes that the binary, with an orbital period of 48 days, is surrounded by a precessing circumbinary disk (e.g., Herbst et al. 2002). Because of the binary motion, the primary and the secondary are being occulted periodically by the disk (e.g., Hamilton et al. 2003; Chiang \& Murray-Clay 2004; Winn et al. 2004; 2006).

Hamilton et al. (2005) measured a rotational period of 9.6 days for the primary star of KH 15D, based on photometric periodicity observed out of eclipse. Assuming pseudo-synchronization, Herbst \& Moran (2005) and Hamilton et al. (2005) derived an eccentricity of $e=0.65 \pm 0.01$. This was probably consistent with the eccentricity of $0.68 \leq e \leq 0.8$, derived by Johnson et al. (2004) from Keck radial-velocity measurements. Indeed, Winn et al. (2006) derived a value of $e=0.574 \pm 0.017$ for the orbit of KH 15D in one of their models. If this value is correct, then the primary star in KH 15D system rotates with a frequency close to its pseudo-synchronization frequency, despite the long orbital period of 48 days. Additional spectroscopic and photometric observations are certainly needed in order to establish the rotational period of the primary, the eccentricity, and then the pseudo-synchronization of this fascinating system.

Recent work on the stellar inclination of $\mathrm{KH} 15 \mathrm{D}$ relative to its orbital motion is reviewed in Section 6.2.3. 


\section{Alignment}

As stated in the introduction, tidal forces in binaries tend also to align the rotation axes of the two stars with the orbital angular momentum. As argued for synchronization, the alignment timescale should be two or three orders of magnitudes shorter than the circularization timescale. This is so because the angular momentum transferred between the orbital motion and the stellar rotation in order to reach alignment is much smaller than that which is needed to attain circularization. This means that we can expect binaries to reach alignment long before reaching circularization, and we expect all circularized binaries to be aligned.

Contrary to circularization and synchronization, we do not know if binaries are formed with misaligned rotation. On the contrary, one could argue that binary systems are formed with stellar spin aligned with the binary motion. This is so because both stellar rotation and orbital motion are relics of the same angular momentum of the primordial cloud, out of which the binary was formed. Common primordial spin-orbit misalignment can rule out some binary formation scenarios (e.g., Durisen et al. 2001; Bate et al. 2002; Bonnell \& Bate 2005; Machida 2005). Thus, we do not know if tidal interaction to attain alignment should set into action at all. It is therefore important for our understanding of binary formation to measure the present extent of the spin-orbit inclinations in the binary population.

Observationally, the spin-orbit inclination, $i_{r e l}$, is difficult to obtain, and consequently not much work has been done on binary alignment. One way to derive the relative inclination is to use stellar spectra with high enough resolution and $\mathrm{S} / \mathrm{N}$ ratio that can yield detailed line profiles. The line profiles can be modelled to give projected stellar rotational broadening $-V_{*} \sin i_{*}$, where $i_{*}$ is the inclination of the stellar rotation axis relative to our line of sight. With an independent knowledge of the size of the stellar radius and rotational period one can estimate the inclination angle $i_{*}$.

Note that this approach allows us to measure the stellar spin inclination relative to our line of sight and not relative to the binary orbit. In order to derive $i_{\text {rel }}$ we also need to know $i_{\text {binary }}$ - the orbital inclination of the binary. This angle is not known for spectroscopic binaries, and can be derived directly from the observations only for eclipsing binaries. The difference between the two inclinations, $i_{\text {binary }}-i_{*}$, is an estimate of the minimum relative angle $i_{r e l}$.

In addition, we do not know the stellar radius and the rotational period for most stars. The stellar radii can be directly obtained only from observations of eclipsing binaries. Estimation of the stellar radii from the spectral type is not very accurate. The stellar rotational period is also not well established, and can be obtained either via special photometric monitoring of the system (see above), or by assuming synchronization. As a result of these drawbacks, the line broadening approach to studying binary alignment was not used extensively in the past. In fact, in some cases the derivation is reversed, and is used to estimate the rotational period (see Section 5). Another example is the work of Beatty et al. (2007), which derived the radius of the primary for the eclipsing single-lined binary HAT-TR205-013 from the observed $V \sin i_{*}$, assuming synchronization and alignment. 
As discussed in Section 3.2, spin-orbit misalignment can cause three noteworthy effects. First, it can change the apsidal precession of the binary by introducing counter apsidal precession. As in the case of DI Her, such an effect can, in principle, be measured if the actual precession is observed and the other precessions can be derived. Second, the binary tidal interaction will cause the stellar spins and the orbital plane to precess around the total angular momentum of the system with the same period. Such a forced precession will change the stellar spin relative to our line of sight, resulting in a change of the stellar rotational broadening of the two stars. Third, it might also slightly change the inclination angle of the binary, which can be detected by radial-velocity measurements, as well as by photometry if the binary is an eclipsing system. However, a study of the old observations of DI Her by Reisenberg \& Guinan (1989) is one of the few works that tried to use this indirect line of evidence for detecting spin-orbit misalignment (see, however, Mayor \& Mazeh (1987) for a different misalignment found in triple systems and the observational search for its effects).

Evidence for significant spin-orbit misalignment should have implications for our understanding of other systems. For example, a forced precession induced by spin-orbit misalignment was already considered in the context of X-ray binaries. It was suggested by Roberts (1974) to account for the 35-day periodicity of Her X-1 (Giacconi et al. 1973), and for the 164-day precession of the relativistic jets of SS 433 (see Margon 1984). This idea was never confirmed for Her X-1, and a different precession model of Katz (1973) became more popular. For SS 433 the evidence is also not conclusive (see a review of SS433 recent observations and theory by Fabrika 2004). Therefore, our comprehension of the typical spin-orbit inclination in binaries is of great importance. It is a pity that the derivation of this inclination from indirect observations is so difficult.

Another, more direct, method to derive the spin-orbit relative inclination angle is through the Rossiter-McLaughlin effect, which is relevant only for eclipsing binaries. This effect is described in detail in the next section.

\subsection{The Rossiter-McLaughlin effect}

The Rossiter-McLaughlin (RM) effect is named after Rossiter (1924) and McLaughlin (1924) who observed the effect for $\beta$ Lyrae and Algol - two famous eclipsing binaries, within the same year.

The RM effect is the varying shape of the stellar line profile during eclipse and is based on a combination of two very basic ideas. The first is stellar rotational broadening, which is due to the fact that different sections of the stellar disk, at different distances from the projected stellar rotational axis, have different Doppler shifts (e.g., Gray 2005). The second is that during the progress of an eclipse, varying parts of the stellar disk of the eclipsed component are covered by the eclipsing star. Therefore, the line shape of the eclipsed star is bound to vary during the eclipse. In fact, the eclipsing body allows us, through observing the RM effect, to indirectly resolve the disk of the eclipsed star. Such an effect was observed, for example, by Rauch \& Werner (2003) in their observations of AA 
Dor.

The RM effect has one additional interesting feature. If the orbital motion of the companion is in the same direction as the stellar rotation, then during the first part of the eclipse the blue-shifted part of the stellar disk is eclipsed, while the red-shifted part of the stellar disk is eclipsed during the second part of the eclipse. If, on the other hand, the orbital motion and the stellar rotation are in opposite directions, then the red-shift part of the disk is eclipsed first. Thus, the RM effect can also detect the relative direction of stellar rotation.

Dividing the eclipse into two periods - one when the red-shifted side of the disc is eclipsed, and the other when the blue-shifted side is eclipsed, might be illuminating on a conceptual level. For example, if the stellar axis is aligned with the orbital motion, then we expect these two parts of the eclipse to be symmetrical for circular orbits. Asymmetry between the two parts clearly indicates a nonzero relative inclination.

The RM effect recently attracted new interest because of its application to extrasolar planets (e.g., Queloz et al. 2000; Winn et al. 2007; Loeillet et al. 2007). However, contrary to the RM effect on a transiting planet, in a binary system the derivation of the relative inclination is not easy, specifically due to the contribution of the secondary star to the total light of the system. In order to follow the distortion of the primary lines when the primary is eclipsed, for example, one must resolve the blending of the primary and secondary lines. This might be complicated whenever the secondary brightness is comparable to the primary brightness, because during the eclipse there is almost no shift between the velocities of the two stars, except for some very eccentric orbits. Such a problem does not exist when analysing the RM effect of transiting planets, as the planet's light is negligible. On the other hand, the amplitude of the effect in a binary is much larger than in that of a planet's transit, as a planet covers during its transit only a very small fraction of the stellar disk of its parent star.

The RM effect can be detected even in spectra taken with low resolution, such that the profile changes can not be resolved. This is done by deriving the stellar radial velocities from the spectra obtained during the eclipse. The velocities are expected to have shifted from their orbital motion value, because the center of gravity of the lines are shifted by the eclipsing component. When the red-shifted part of the stellar disk is eclipsed the velocity is shifted to the blue and vice versa. The amplitude of the shift depends on the relative spin-orbit inclination, but also on the rotational velocity of the eclipsed star, the ratio of the two radii and the limb darkening of the stellar disk (Kopal 1942; 1959; Hosokawa 1953; Ohta et al. 2005; Winn et al. 2005; Giménez 2006).

In fact, almost all observations of the RM effect were performed by observing the distortion of the radial-velocity curve during the eclipse. This includes the original work of Rossiter and McLaughlin, and the more recent work of Worek (1996), who observed AI Dra and V505 Sgr. In the next subsection we review two additional interesting observational studies of DE Dra and V1143 Cyg. 


\subsection{Interesting cases}

\subsubsection{DE Dra}

DE Dra is an eclipsing single-lined spectroscopic binary (Furtig \& Meinunger 1976), consisting of a B9V primary in a 5.3 day circular orbit (Hube 1976). Hube \& Couch (1982) obtained many spectra of the system during and outside the eclipse, in order to derive the radial-velocity modulation of the system. The obtained radial velocities clearly showed the RM effect during eclipse. The distortion of the radial-velocity curve was significantly asymmetric, suggesting a spin-orbit inclination of the primary. The analysis of Hube \& Couch of the distorted radial-velocity modulation indicated a rotational broadening of $180 \mathrm{~km} \mathrm{~s}^{-1}$. They performed an independent line profile analysis which yielded a rotational velocity of only 135 $\mathrm{km} \mathrm{s}^{-1}$. It seems as if the inconsistency between these two values prevented Hube $\&$ Couch from carrying their analysis of the RM effect further and deriving a firm value for the relative inclination of the primary axis.

The observations and analysis of Hube \& Couch (1982) were not performed with modern techniques, so the statistical significance of their results is questionable. However, if true, this is the only binary that we know of that has a non-aligned rotational axis. This is most interesting because it can tell us something about the binary formation of DE Dra. In fact, the system has another feature which indicates that the binary has not yet reached tidal equilibrium. The primary rotational velocity, being either 180 or $135 \mathrm{~km} \mathrm{~s}^{-1}$, is spinning much faster than the synchronization velocity, which is about $30 \mathrm{~km} \mathrm{~s}^{-1}$. Therefore, we probably have in hand a binary system that has completed its circularization, if it was formed with eccentric orbit, but has not yet reached synchronization and alignment.

The exceptional features of this system make further observations and analysis very desirable. New observations can confirm the non-alignment of the primary, resolve the secondary spectrum, study the secondary eclipse, and search for forced precession of the primary, which should manifest itself in varying inclination of the stellar axis. The latter feature would add new information that would contribute to our understanding of this remarkable system.

\subsubsection{V1143 Cyg}

Just before the conclusion of this manuscript, Albrecht et al. (2007) put a beautiful paper in astro-ph, which reports on a very careful study of the RM effect in the eclipsing binary V1143 Cyg. This binary (see Section 5.3.1) consists of two F5V stars orbiting each other with a period of 7.6 days and an eccentricity of 0.54 . As stated above, it is one of the very few binaries of which we know from observations that one of the components reached pseudo-synchronization for a highly eccentric orbit. However, only the secondary reached pseudo-synchronization, while the primary is still rotating with a period which is larger by $50 \%$ than the pseudosynchronization period (Andersen et al. 1987). The fact that the apsidal motion of the system (Giménez \& Margrave 1985) might be slightly smaller than the theoretical expectation (see discussion above in section 3.2 for DI Her) motivated 
Albrecht et al. (2007) to study the relative inclination of the two stars through the RM effect.

Analysing high $\mathrm{S} / \mathrm{N}$ ratio spectra in detail, Albrecht et al. derived the angle $\beta$ between the stellar spin axis and the orbital angular momentum, both projected onto the plane of the sky. Obviously, $\beta=0$ is consistent with perfect spin-orbit alignment. They found that $\beta_{A}=0.5 \pm 4^{\circ}$ and $\beta_{B}=-3.9 \pm 4^{\circ}$, for the primary and the secondary, respectively, to be consistent with a perfect alignment of both components.

These results are most interesting, showing that the primary is aligned despite the fact that the binary is not synchronized. We would like to suggest that this might indicate that the V1143 Cyg system was formed with a rotation axis aligned with the orbital binary motion, at least for the primary. This is so because we assume that the timescale for pseudo-synchronization and alignment are similar, and therefore the non-synchronization of the primary indicates that there was not enough time during the lifetime of the system for the primary to reach synchronization or alignment. If this is true, observing the RM effect of V1143 Cyg told us something about the formation of the binary instead of its tidal evolution.

\subsubsection{KH $15 \mathrm{D}$}

KH $15 \mathrm{D}$ is a T Tau binary with a period of 48 days, which is surrounded by a precessing circumbinary disk (e.g., Herbst et al. 2002). The primary and the secondary are being occulted periodically by the disk (for a more detailed account of this interesting system see Section 5.3.2).

Winn et al. (2006) pointed out the possible RM effect induced by the disk when occulting the visible star of KH 15D or its halo. However, the optical spectra they obtained did not allow a quantitative analysis of this effect. Therefore, there is no information available for alignment or misalignment of the primary of $\mathrm{KH} 15 \mathrm{D}$ relative to its binary orbit. However, strong evidence was found for an inclined, warped, eccentric, precessing circumbinary disk (Chiang \& Murray-Clay; Winn et al. 2004; 2006) with a precession period of the order of 1000 years. The cause of the misalignment between the binary plane of motion and its circumbinary disk is still a mystery, and might indicate some basic misaligned component of the angular momentum left from the formation era of the system.

\section{Tidal Interaction in Close Triple System}

As stated in the introduction, tidal interaction in close binaries acts until the binary reaches an equilibrium state, in which the orbit is circularized, the stellar rotations are synchronized and the stellar spins are aligned with the orbital motion. It is only when this state is reached that the stellar tides do not move relative to the surfaces of the two stars, and the stellar shapes are constant in the rotating frame of reference.

However, when a binary is a part of a hierarchical triple system, which is composed of the binary and a third distant companion, such a stable configuration 
might not be possible. The reason for this is that the third star constantly injects eccentricity into the binary orbit by exerting its own 'tidal forces' on the binary system as a whole. The origin of these tidal perturbations is the small difference between the gravitational acceleration of the two individual stars induced by the third star and the corresponding acceleration of the center of mass of the binary system. This difference does not depend on the actual radii of the two stars, but instead depends on the size of the binary system relative to its distance from the third star.

One can classify the perturbation of the third star into three different effects.

- Modulation of the binary eccentricity.

- Apsidal precession of the binary.

- Nodal precession of the binary orbit around the total angular momentum of the triple system.

The effects of the third star can appear on three timescales: the binary period, the third star period, and a long-term modulation (e.g., Brown 1936; Borkovits \& Forgács-Dajka 2008, this volume). In what follows we are mainly interested in the long-term modulation and its interrelation with the long-term tidal interaction that is occurring in the binary.

It is advisable that one take the possible effects of an unknown distant companion into consideration when observing binary stars, because a third distant companion could easily escape the notice of observers in the course of studying binary systems. Unfortunately, in many cases no effort is made to search for a faint, distant companion after a binary is discovered and studied. Nevertheless, several lines of evidence indicate that the percentage of binaries that have a distant third companion is significant.

Mayor \& Mazeh (1987) were the first to search systematically for third companions of spectroscopic binaries, using the precession of the nodes induced by the third companion. They looked in a small sample of spectroscopic binaries and found evidence that $25 \%$ of the members of their sample have third companions. Other studies (Isobe et al. 1992; Tokovinin \& Smekhov 2002; Pribulla \& Rucinski 2006; D'Angelo et al. 2006) also indicated a high frequency of triple systems. A recent search by Tokovinin et al. (2006) found that $96 \%$ of binaries with periods shorter than 3 days have a third companion! A major step forward in the observational study of triple systems is the construction of a new catalog by Tokovinin which includes all known stellar triple systems (1997; see also the on-line version at http://www.ctio.noao.edu/ atokovin/stars/index.php).

Note that the nodal precession of the orbital plane of a binary caused by a third star, an effect used by Mayor \& Mazeh (1987) to search for triple systems, might induce spin-orbit misalignment into the binary system (Fabrycky \& Tremaine 2007)1. Therefore, observational evidence for such misalignment might

\footnotetext{
${ }^{1}$ I thank D. Fabrycky for pointing this point to me.
} 
suggest a third companion. Spin-orbit misalignment due to a third star was suggested by Beust et al. (1997) for the eclipsing binary TY Cor Aus.

This section will review the theory of long-term binary eccentricity modulation and apsidal motion induced by a third star and will point out its application for the tidal evolution of binaries that reside in triple systems. In fact, as suggested already by Mazeh \& Shaham (1979) and now supported by the observations and statistical analysis of Tokovinin et al. (2006), a third companion might have a major role in the formation of very short binaries through constant pumping of the binary eccentricity.

\subsection{The binary modulations induced by a third distant companion}

The zero-order approximation of the dynamics of hierarchical triple stellar systems assumes that the close binary moves in an orbit with its own semi-major axis $a_{1,2}$ and period $P_{1,2}$, while the third distant companion moves together with the binary center of mass in an orbit with $a_{3}$ and $P_{3}$. The hierarchy of the system relies on the ratio $a_{1,2} / a_{3}$ being small. Harrington $(1968 ; 1969)$ developed a theory of hierarchical triple stellar systems by expanding their Hamiltonian into a series of terms in the small parameter $a_{1,2} / a_{3}$. In order to obtain the long-term modulation, Harrington further averaged the Hamiltonian over the binary and the third star periods. The resulting Hamiltonian showed that the tidal forces of the third star vary the binary eccentricity $e_{1,2}$, the longitude of the periastron $g_{1,2}$ and the direction of the plane of motion with quasi-periodic modulation. The modulation period is of the order of $T_{m o d}$ :

$$
T_{\text {mod }}=P_{1,2}\left(\frac{a_{3}}{a_{1,2}}\right)^{3} \frac{M_{1}+M_{2}}{M_{3}},
$$

where $M_{1}$ and $M_{2}$ are the masses of the two components of the binary and $M_{3}$ is the mass of the distant companion (Mazeh \& Shaham 1979). One can then derive the equation of the binary eccentricity:

$$
\frac{d e_{1,2}}{d t}=\frac{2 \pi}{T_{m o d}} \gamma \sin \left(2 g_{1,2}\right) e_{1,2},
$$

where $\gamma$ is a geometrical factor of order unity (Mazeh \& Shaham 1976; 1979). This equation conjugates the binary eccentricity variability with that of the longitude of the periastron, which is also being varied by the third star:

$$
\frac{d g_{1,2}}{d t}=\frac{2 \pi}{T_{\text {mod }}} \gamma\left[\delta+\cos \left(2 g_{1,2}\right)\right],
$$

where $\delta$ is another geometrical constant of order unity. (For a different approach to the derivation of the apsidal motion induced by a third star see, for example, Brown 1936; 1937). The two equations consider the three stars as point masses and ignore general relativistic effects.

Further studies of the dynamics of triple stellar systems as three mass points include the work of Krymolowski \& Mazeh (1999) and Ford et al. (2000; 2004) who 
expanded the Hamiltonian to higher orders of $a_{1,2} / a_{3}$ and that of Beust (2003), who applied a symplectic integration to the stellar triple problem.

In any specific triple system, the actual binary apsidal motion includes the precession induced by the binary tidal interaction and the general relativistic effect (see Section 3). Bozkurt \& Değirmenci (2007) have just recently published a study of the apsidal motion of several known triple systems with detected apsidal motion. However, for all the systems they considered, the apsidal motion induced by the third star is several orders of magnitude slower than the observed one. The reason for this is that in all systems considered by Bozkurt \& Değirmenci, the existence of the third star was discovered by timing the eclipses minima and using the lighttravel time technique. In principle, this is the same technique as that by which the apsidal motion was detected. Therefore, the orbital period of the third star, $P_{3}$, is necessarily of the same order of magnitude as the detected apsidal motion. Consequently, the apsidal motion induced by the third star is much longer than the detected one. This can be seen by writing Equation (7.1) as

$$
T_{\text {mod }} \simeq P_{3}\left(\frac{P_{3}}{P_{1,2}}\right) \frac{M_{1}+M_{2}}{M_{3}},
$$

which implies that $T_{m o d}$ is much longer than $P_{3}$. In order to find triple systems with shorter apsidal binary motion, one need to look for triple systems with a much smaller $P_{3} / P_{1,2}$ ratio (see Tokovinin (1997) catalog of multiple systems). Two such systems are G:38-13 (Mazeh et al. 1993) and HD 109648 (Jha et al. 2000). In these systems the period of the modulation induced by the third star is of the order of 100 years.

\subsection{The eccentricity modulation combined with tidal circularization}

In many triple systems, $T_{\text {mod }}$ is of the order of the circularization timescale, $\tau_{c i r c}$, or shorter. In such cases one must take the modulations induced by the third star into account when considering the tidal evolution of close binaries, particularly their eccentricity.

Mazeh \& Shaham (1979), for example, considered the long-term effect of the eccentricity modulation induced by the third star together with the binary tidal circularization (Equation 4.2)

$$
\frac{d e_{1,2}}{d t}=-\frac{1}{\tau_{\text {circ }}} e_{1,2},
$$

which is also linear in $e_{1,2}$, and got, to first order, a periodic modulation combined with an exponential decay, as displayed schematically in Figure 5 .

Figure 5 presents a moderate third-star modulation of the eccentricity. However, this is not necessarily the case for all systems, as it all depends on the relative inclination of the two orbits. As pointed out by many studies (e.g., Krymolowski \& Mazeh 1999; Ford et al. 2000; 2004) the third star 'tidal forces' can inject a high eccentricity into the binary motion, if the inclination angle of the plane of 


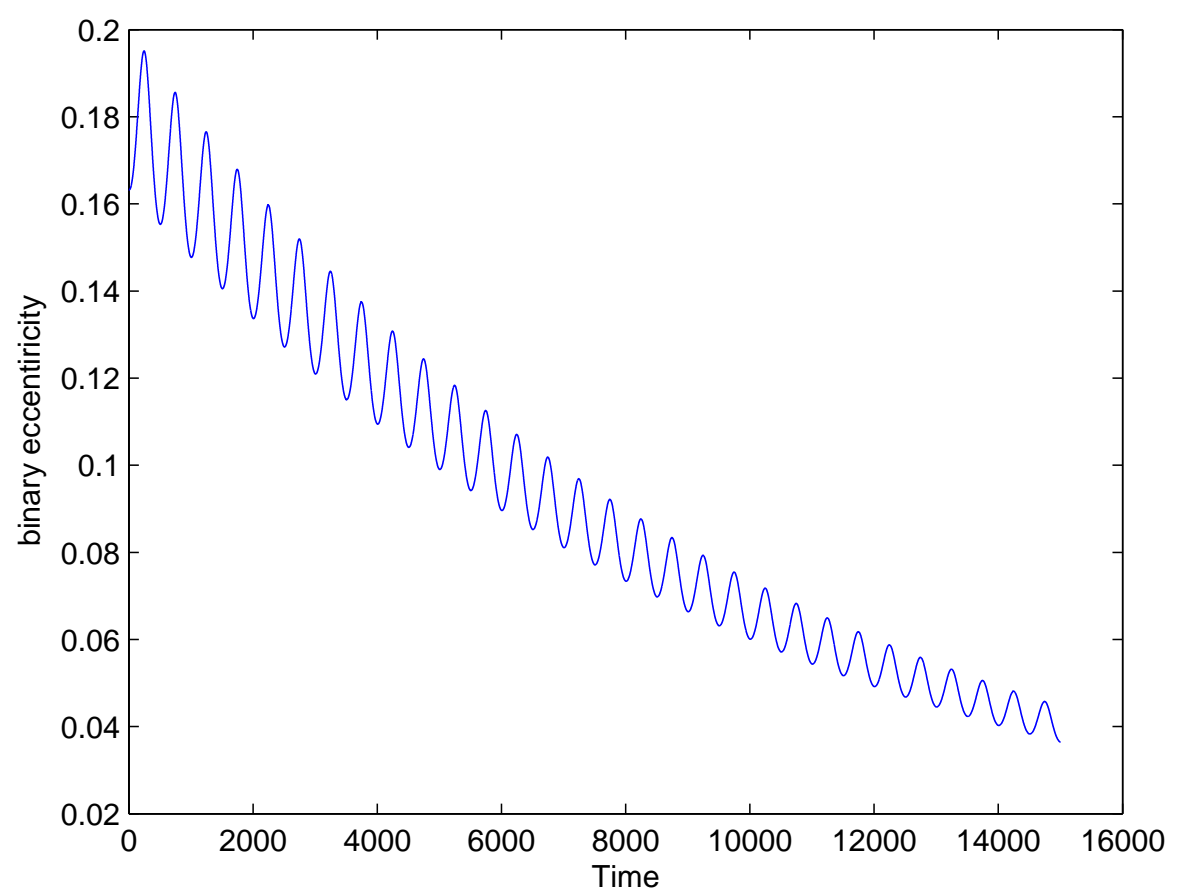

Fig. 5. Schematic presentation of the eccentricity evolution of a binary that resides in a triple system, after Mazeh \& Shaham (1979).

motion of the third star relative to the binary plane of motion is higher than approximately $40^{\circ}$. This high-amplitude modulation was first noted by Kozai (1962) for the motion of asteroids and was first applied to stellar systems by Mazeh \& Shaham (1976), who at that time were unaware of Kozai's work.

As the third star modulation and the tidal circularization are both linear in $e$, one could conclude that at the end of the circularization process the modulation decays to zero. However, Mazeh and Shaham (1979) have demonstrated that the linearity of the third star modulation is true only to the first order of $a_{1,2} / a_{3}$. Upon integrating Newton's equations directly, they discovered that the eccentricity modulation is present even if the binary eccentricity starts with zero. The Mazeh \& Shaham effect was further theoretically studied and confirmed by Georgakarakos (2002; 2004). We therefore expect some short-period binaries that should have been circularized long ago to display small eccentricities.

In fact, observational evidence for such an effect has already been pointed out by Mazeh (1990) in three short-period binaries, with significant eccentricities and strong indications for a third distant stellar companion. One of the examples found by Mazeh is the Hyades star BD $+23^{\circ} 635$, which has an orbit of 2.4 days and an 
eccentricity of $0.057 \pm 0.005$ (Griffin \& Gunn 1981). Giuricin et al. (1984) also suggested that the small but significant eccentricities they found in systems with fractional primary radius larger than 0.3 (see Section 4.3 for details) must be due to unknown third distant companions. In all these cases, the tidal processes should have circularized the orbits long ago, and the interaction with the third star seems to be the only plausible conjecture that accounts for the observed eccentricity.

The discovery of Mazeh \& Shaham (1979) that the binary eccentricity induced by the third star continues its modulation even after the complete tidal circularization may have implications for the evolution of close binaries. The eccentricity modulation implies that binaries with close enough companions will never get into a stable circular orbit, which means that the binary tidal dissipation will continue to work forever. The injected binary eccentricity invokes frictional forces inside the two stars that dissipate rotational energy and transfer angular momentum between the stellar rotation and binary orbital motion. This causes the semi-major axis of the binary to decrease at a rate of

$$
\frac{1}{a_{1,2}} \frac{d a_{1,2}}{d t}=-\frac{1}{T_{\text {circ }}} A e_{1,2}^{2}
$$

where $A$ is a geometrical factor of order unity. Mazeh and Shaham (1979) suggested, therefore, that in certain configurations the third distant companion can provoke the spiral-in of the close binary at a rate that can be derived by averaging Equation 7.6. The third star motion can serve as the sink for the angular momentum of the shrinking binary.

Obviously, the separation of the perturbations of the third star and the tidal friction into two equations is artificial, and was done only as a first approximation. Further studies, including Kiseleva et al. (1998), Eggleton \& Kiseleva-Eggleton (2001; 2006), Eggleton (2006), Borkovits et al. (2004; 2005; 2007) and Fabrycky \& Tremaine (2007) combined the two effects into one set of coupled equations. Kiseleva-Eggleton, Eggleton and Fabrycky \& Tremaine further studied the Mazeh \& Shaham effect for cases where the eccentricity modulation is large, which occurs when the relative angle between the two orbital planes of motion is larger than $\sim 40^{\circ}$. They also concluded that some of the presently close binaries could have been formed by the combination of tidal binary friction and the third star induced modulation. The Tokovinin et al. (2006) result, that $96 \%$ of binaries with periods shorter than 3 days have a third companion, might be a strong evidence for the Mazeh \& Shaham conjecture.

\section{Extrasolar planets and tidal Interaction}

Since the discovery of 51 Peg b (Mayor \& Queloz 1995), more than 200 planets have been discovered (see, for example, the Extrasolar Planets Encyclopaedia22). As one can consider a star with a planet as a binary with an extremely small

${ }^{2}$ http://vo.obspm.fr/exoplanetes/encyclo/catalog-RV.php 
mass ratio, we can apply the theory of tidal interaction to these systems, and therefore expect to find some signs of its action. In particular, we expect the planets with short enough orbital periods to attain circularization, alignment, and planetary synchronization. This section will review in brief some observational lines of evidence that might indicate that tidal interaction between extrasolar planets and their parent stars took place during the lifetime of those systems.

\subsection{Radial drift by tidal interaction}

One possible outcome of the tidal interaction between a planet and its parent star is a radial drift of the planet, resulting from the transformation of angular momentum between the stellar rotation and the planetary orbital motion. The angular momentum of the planetary orbital motion is proportional to $m_{p} \sqrt{a_{p}}$, where $a_{p}$ is the planetary orbital radius and $m_{p}$ is the planetary mass, and is small relative to the stellar angular momentum for planets with orbital periods of a few days. Therefore, transfer of a small amount of angular momentum from or into the planetary orbital motion can substantially change the planetary orbital radius, an effect which is unique to planetary motion. This subsection discusses the two possible directions of the radial drift and their astrophysical implications.

\subsubsection{Stopping the migration}

One possible effect of the radial drift was suggested already by Lin et al. (1996) for $51 \mathrm{Peg} \mathrm{b}$, whose orbital radius is about $0.05 \mathrm{AU}$. The idea is based on the paradigm that Jupiter-sized planets can not be formed close to their parent stars, at a distance of $\sim 0.05 \mathrm{AU}$, but instead must be formed behind the 'ice line' of the protoplanetary disk, at a distance of $\sim 5 \mathrm{AU}$ (e.g., Bodenheimer \& Pollack 1986; but see, however, Boss 1997 for a heretical approach). At that distance the icy particles initially form a rocky core of several earth masses, which subsequently accretes gas to form a giant planet. In order to save the core-accretion model, Lin et al. suggested that $51 \mathrm{Peg} \mathrm{b}$ migrated from the location of its formation into its present radius through interaction with the material in the disk, an idea that had already been discussed a decade before the discovery of 51 Peg b (e.g., Goldreich \& Tremaine 1980; Papaloizou \& Lin 1984; Lin \& Papaloizou 1986) in the context of the solar system. Such a model for the short-period extrasolar planets had to explain why the migration stopped at $\sim 0.05 \mathrm{AU}$ and did not push the planet into the surface of the star. The idea of Lin et al. was that this was done by the tidal interaction between the planet and its parent star.

This 'stopping mechanism' can work only if the star rotates on its spin axis faster than the orbital frequency of its planet. In such a case, the tidal interaction between the planet and the star tends to slow down the stellar rotation, taking angular momentum from the star into the planetary orbital motion. The additional orbital angular momentum forces the planetary orbital radius to increase. This result is counterintuitive, because the tidal interaction eventually slows both the planetary orbital motion and the stellar rotation. Nevertheless, it relies on 
the conservation of angular momentum and Kepler's laws, and must therefore be correct.

According to this scenario, the planet can migrate inwards as long as the interaction with the disk is stronger than the stopping force of the tidal interaction with the star. The planet stops its migration when the two forces cancel each other out. The timescale of the radial drift caused by the interaction with the spinning star was estimated by Lin et al. (1996) to be:

$$
\tau_{\text {radial }}=\frac{a_{p}}{\left|\dot{a}_{p}\right|}=\frac{9}{2 n_{p}} q^{-1}\left(\frac{a_{p}}{R_{*}}\right)^{5} Q_{*} \propto \frac{M_{*}^{1 / 2} Q_{*}}{R_{*}^{5}} \frac{a_{p}^{13 / 2}}{m_{p}}
$$

where $n_{p}$ is the Keplerian angular velocity of the planet, $q=m_{p} / M_{*}$ is the mass ratio between the planetary mass, $m_{p}$, and the stellar mass, $M_{*}, R_{*}$ is the stellar radius, $a_{p}$ is the planetary orbital radius and $Q_{*}$ is the stellar tidal dissipation factor (See Sasselov et al. 2003 for a different expression of $\tau_{\text {radial }}$ ). Note that Trilling et al. (1998) pointed out that the numerical coefficient in this equation is only true for bodies of uniform density, and should be corrected for the actual stellar mass profiles.

The problem with this equation is the unknown factor $Q_{*}$, which describes how efficiently rotational energy is dissipated by friction within the star. Lin et al. (1996) adopted a value of $1.5 \times 10^{5}$ "for a main-sequence star, based on the observations that the orbits of short-period pre-main-sequence binary stars and the main-sequence binary stars in the Pleiades cluster are circularized for periods up to 5 and 7 days, respectively". We note, however, that the error on this derivation of the value of $Q_{*}$ from the transition period of the Pleiades could be substantial for two reasons. First, the binaries in the Pleiades cluster might have been circularized during their pre-main-sequence phase. In such a case, we can not use the transition period of the Pleiades at all. Second, we can not estimate the exact transition period, nor do we know the exact mass of the secondaries in the binaries of those clusters, as was discussed in Section 4.4.1 in detail. The factor $Q_{*}$ is also a strong function of the star itself, depending on the depth of the convective envelope and the stellar radius at the time of the inward migration (e.g., Sasselov 2003).

As we can see from Equation 8.1, the stopping mechanism is a strong function of the planetary orbital radius, and the radial-drift timescale drops dramatically for small distances. The equilibrium between the interaction with the disk and the tidal interaction with the spinning star depends on the disk mass and density profile on the one hand, and the stellar structure and the intensity of the stellar interaction on the other hand. According to this scenario, when the protoplanetary disk disappears, the planet will drift outward until its radial-drift timescale gets too long.

Some support for the stopping mechanism scenario can be found in the period distribution of the known planets, which is plotted in Figure 6 (Data were taken 




Fig. 6. The period distribution of the extrasolar planets.

from the Extrasolar Planets Encyclopaedid 3). One possible interpretation of the figure is that between 2 and 200 days the distribution is flat in log period, with an excess of about 20 planets around the period of 3 days. According to the migration scenario and its stopping extension, at least half of the planets with periods of around 3 days were stopped by the tidal interaction during their inward migration. The excess of planets with periods around 3 days is still there even if we take out the transiting planets, for which there is a strong selection effect at short periods.

This interpretation of the observed distribution implies that many of the parent stars of the planets with orbital periods of 3 days have, or at least had in the past, rotational periods shorter than 3 days. We can try to corroborate this conjecture by deriving the present stellar rotational periods of these systems. One approach to deriving the rotation period is based on the rotational broadening of the stellar profiles, relying on the stellar radius and inclination. However, even if we know the inclination, this approach is quite inaccurate, as discussed in Section 5

Another approach is to follow the photometric variation of the parent stars

${ }^{3}$ http://vo.obspm.fr/exoplanetes/encyclo/catalog-RV.php 
that can reveal their rotational periods. Consider, for example, the M-type star Gliese 436, whose planet was discovered by Butler et al. (2004), and its transiting nature was revealed only recently by Gillon et al. (2007). Demory et al. (2007) found some evidence for a photometric modulation with a period of about 45 days, which could be the rotational period of the parent star. If this is true, then the rotational period of the $\mathrm{M}$ star is much longer than the 2.6 day orbital period of the planet. Bonfils et al. (2007) found a similar situation for Gliese 674, for which the orbital period is 4.7 days and the rotation period of the $\mathrm{M}$ star is about 35 days. However, the assumed long rotational periods of these two stars could be the result of spinning down of the stellar rotation, a commonly observed phenomenon, in M-type stars in particular (e.g., Cardini \& Cassatella 2007).

Other mechanisms can be invoked to explain the peak of the period distribution of the extrasolar planets at around 3 days. For example, one can assume that the protoplanetary disks have been evaporated when the planets arrived into their present radii, which correspond to periods around 3 days. After all, we know that the disks disappear after a period of the order of 10 Myrs, either by stellar wind and photoevaporation or by viscous processes in the disk itself (e.g., Haisch et al. 2001). Another version of this stopping mechanism was suggested by Kuchner \& Lecar (2002), who assumed a central 'hole' in the protoplanetary disks, with an edge corresponding to an orbital period of 6 days. The central hole is formed by turbulent accretion due to magnetorotational instability that acts on the material in the central part of the disk, where small grains reached sublimation temperature. The planetary migration halts when the exterior 2:1 Lindblad resonance reaches the external radius of the hole. Another stopping mechanism (e.g., Trilling et al. 1998) is based on the assumption that the short-period planets got so close to their parent stars that their Roche Lobe became smaller than their planetary radii. This caused mass transfer from the planets onto the stars, which resulted in an outward motion of the planet in order to conserve angular momentum.

A different idea was raised recently by Burkert \& Ida (2007), who suggested that the period distribution peak was in fact a pronounced dip at the range of 10-100 days. Furthermore, their analysis indicated that the dip appeared only in planets around F-type stars, with masses larger than $1.2 M_{\odot}$, while the distribution of planets around G-type stars showed log-flat distribution. To account for the different period distributions, Burkert \& Ida assumed that the sizes of the protoplanetary disks around $\mathrm{F}$ and $\mathrm{G}$ stars were different, resulting in different patterns of migration. Eventually, this caused a gap in the period distribution of planets around F stars, but not around G stars. It seemed that their simulations could produce the period distribution of the planets and did not necessitate an extra stopping mechanism.

We note that the distinction between the period distribution of planets around $\mathrm{F}$ and $\mathrm{G}$ stars is an interesting feature of the planet population, and might be accounted for by different tidal interactions with the parent stars, instead by the different masses of the disks. This is so because tidal interaction in $\mathrm{F}$ stars is substantially weaker than in $\mathrm{G}$ stars, due to the fact that $\mathrm{F}$ stars do not have convective envelopes. Thus, F stars can stop the inward migration only when the 
planet is substantially closer to the parent star than the stopping radius of G stars. Consequently, if tidal interaction with their parent stars plays a role in the final orbits of planets, the difference in the period distribution of planets around $\mathrm{G}$ and F stars could have been caused by internal stellar structure differences.

The present discussion clearly demonstrates that the extra planets at an orbital period of around 3 days are still not acceptable evidence for the action of tidal interaction between short-period planets and their parent stars, and that further observations and theoretical work are still necessary.

\subsubsection{Inward drift}

Tidal interaction can also push the planet inwards, if the planet orbital frequency is faster than the stellar spin. In such a case, the planet is dragged back as a result of tidal interaction with the star, an interaction that takes angular momentum from the planetary orbital motion, which results in a decrease in the orbital radius of the star. The timescale for the inward drift is given by Eq. 8.1 that describes the outward drift, but now $\dot{a}_{p}$ is negative. However, unlike the outward drift, the inward drift is a runaway process, in which $a_{p}$ gets smaller and therefore the drift timescale, $\tau_{\text {radial }}$, gets shorter, which accelerates the drift further (e.g., Rasio \& Ford 1996).

It is therefore convenient to define the corotation radius, in which the planetary orbital period equals the stellar rotational period. Outside this radius the planet is pushed outward, while within this radius the planet is pushed inward. If interaction with the disk succeeds in pushing the planet inside the corotation radius, then tidal interaction with the star joins forces with the disk interaction to push the planet inward. The planet can survive inside its corotation radius only if the disk disappears and the drift timescale is long enough. Rasio \& Ford concluded that for $51 \mathrm{Peg}$ the timescale for the inward drift is shorter than the stellar lifetime only if the orbital period is shorter than about 10 hours. This means that we would not be able to find planets around stars similar to $51 \mathrm{Peg}$ with orbital periods shorter than 10 hours, which indeed is the case.

We note that the stellar radii and rotational periods do change during the stellar lifetime, and both the corotation radius and the radial-drift timescale necessarily follow these variations. As the stellar rotational period gets longer and the corotation radius increases, the planet can find itself inside the corotation radius, being pushed inwards by the tidal interaction with its parent star. Such a planet can survive only if its orbital radius is large enough so the drift timescale is longer than the stellar lifetime.

As of November 2007, eleven planets were detected with periods shorter than 2 days, the shortest of which is OGLE-TR-56b, with a period of 1.2 days (Konacki et al. 2003). The discussion above highlights the need to carefully consider the stability of the orbits of these planets against inward drift. Sasselov (2003), for example, tried to exclude some versions of the tidal theories that predict too fast radial drift for OGLE-TR-56b. He suggested that for some theories it would be possible to detect in the near future changes in the orbit of OGLE-TR-56b by 
precise timing of its transit. Such a measurement would be a landmark of the observational evidence for tidal interaction in main-sequence stars, enabling us to follow the results of the interaction in real time, and not only collect indirect proofs for its action in the past.

\subsection{Circularization of extrasolar-planet orbits}

In order to study the tidal circularization of extrasolar planets, we plotted in Figure 7 the eccentricities versus periods of all known planets, like we did in Figure 1 for all the known spectroscopic binaries. The main two features of Figure 1 can also be found in Figure 7 (see Halbwachs et al. 2005 for a detailed comparison). All planets with periods shorter than a 'cutoff' period are circular, and all the extrasolar planets with longer periods have eccentricities below an 'upper envelope', which starts at eccentricity zero and climbs up asymptotically to a certain high value. The values of the planetary upper envelope parameters are different from those of the spectroscopic binaries. The upper envelope starts to rise at a period of 2.5 days and rises to an eccentricity of 0.8 when moving towards 25 days.

Figure 7 displays only three exceptions to these two features. Two of them are the very highly eccentric planets HD 80606b (Naef et al. 2001) and HD 20782b (Jones et al. 2006), both with an eccentricity of 0.92 , and the third one is HD $41004 \mathrm{Bb}$ - a planet with one of the shortest periods, 1.3 days, with a small but significant eccentricity of $0.081 \pm 0.012$ (Zucker et al. 2003; 2004). Note that the three eccentricities would not be considered exceptions for spectroscopic binaries, because the upper envelope for binaries reaches a value of 0.98 , and the 'cutoff' period is as short as 0.35 days.

HD 41004 is an interesting system which is composed of a double visual star, HD 41004 A and B, separated by about 20 AU. Each of the two stars has a lowmass companion. Component A has a planet with a period of about 1000 days, with a minimum mass of 2.5 Jupiter mass $\left(=M_{J}\right)$, whereas component $\mathrm{B}$ has a companion with a short period and a small but significant eccentricity, with a minimum mass of $18 M_{J}$ (Zucker et al. 2004). We note that the general consensus puts the upper-mass limit for planets around 10-15 $M_{J}$, and therefore it is not clear if HD $41004 \mathrm{Bb}$ is a planet, or rather a low-mass companion which should be referred to as a brown-dwarf secondary. If HD $41004 \mathrm{Bb}$ is a brown-dwarf companion, it should be moved from Figure 7 to Figure [1] where its location is below the upper envelope. Another possibility is that the visual companion, HD 41004A, pumped eccentricity into the orbit of Bb, as is discussed regarding triple systems in Section [7.1. A similar idea was already proposed for another high eccentricity planet, 16 Cyg B (Mazeh et al. 1997; Holman et al. 1997). For a review of this idea see Mazeh (2007).

The two prevailing features of Figure 7 might be attributed to tidal circularization acting on the extrasolar planets, exactly as the general consensus interprets Figure 1. However, concealed behind the model for the eccentricity of the spectroscopic binaries is the assumption that all binaries, without any period difference, have been formed with the same eccentricity distribution, including the highly 


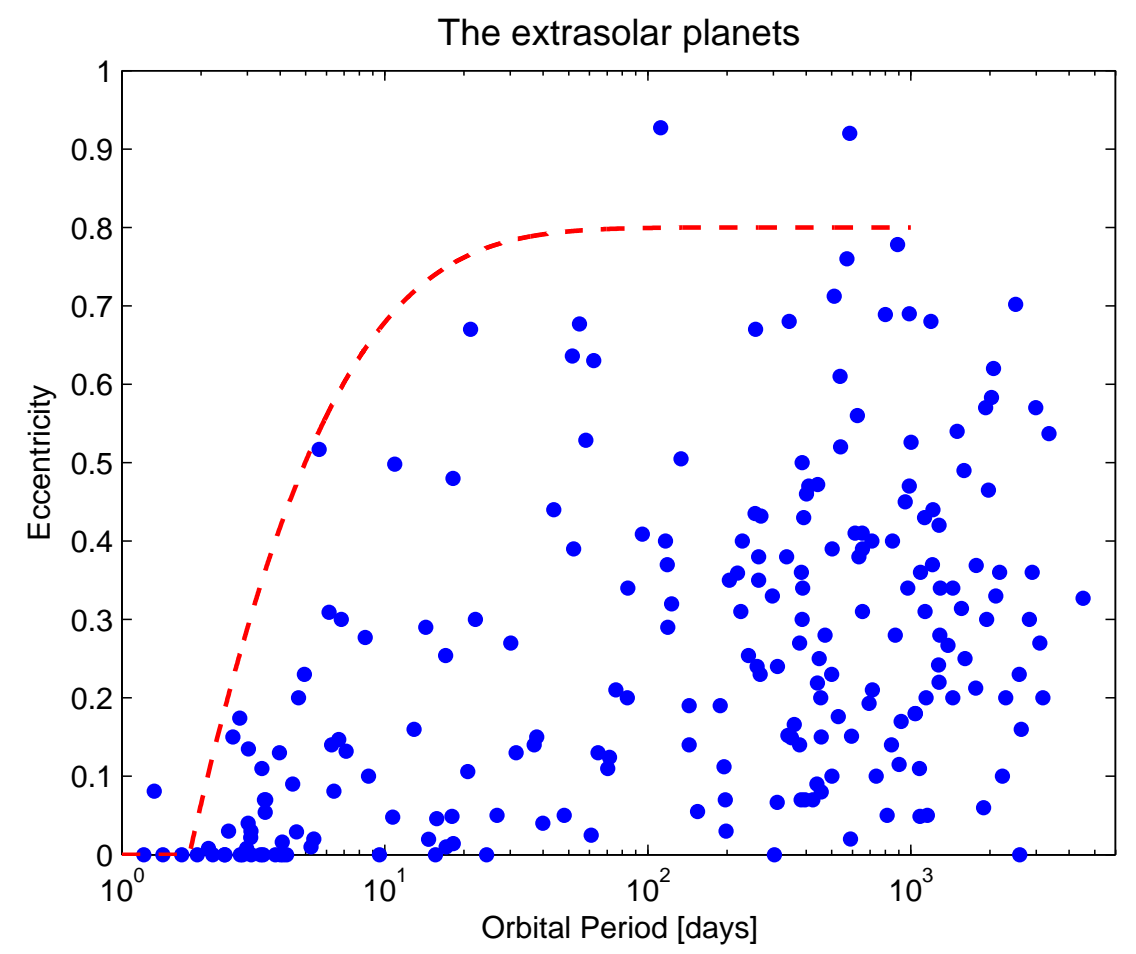

Fig. 7. The eccentricity of the extrasolar planets as a function of the orbital period. The dashed line is the upper envelope, with an equation $f=E-A \exp \left(-(p B)^{c}\right)$, where $E=0.8, A=8, B=6$ and $C=0.35$.

eccentric binaries. Therefore, the fact that short-period binaries are all circular must be the result of some circularization processes occurring after the binary formation. On the other hand, the prevailing assumption for planets was, until just a few years ago, that all planets should have circular or at least almost circular orbits, like all the planets in our solar system. If planets are formed in an accretion disk, then the 'natural' outcome is planets with circular orbits, as the interaction of the planetesimal with the gas and dust in the disk should dissipate any initial eccentricity. This is one of the reasons why HD114762b, detected already in 1989 (Latham et al. 1989) with an eccentricity of 0.4, was not considered as a planet candidate at that time. Therefore, the discovery of planets with large eccentricities was a surprise to the astronomical community. Thus, theoretical effort was not devoted to explaining the circular short-period planets, but instead to building a reasonable scenario for the unexpected eccentricities of the long-period planets.

Some studies associated the observed eccentricities of extrasolar planets with another surprising feature of the planets - their small semi-major orbital axis. As discussed in the previous subsection, the assumed paradigm to explain the short 
distances of the short-period extrasolar planets is an inward migration driven by interaction with the disk. The effect of this interaction on the planetary orbital eccentricity (e.g., Goldreich \& Sari 2003) is still a matter of controversy (see discussion and short review by Moorhead \& Adams 2007). Other studies tried to explain the existence of short-period planets and the planetary eccentricities as a consequence of interaction with other, still undetected, planets (e.g., Weidenschilling \& Marzari 1996; Rasio \& Ford 1996; Zhou \& Lin 2007; Juric \& Tremaine 2007). It is not clear if these scenarios could explain the distribution of eccentricities in long-period planets and the circular orbits of short-period planets at the same time. If this is not possible, and the assumed mechanism that pumped eccentricities into the long-period orbits also necessarily built high eccentricity in some of the short-period planets, then we might need to invoke tidal circularization to account for the circular orbits of the short-period planets.

The circularization of a planetary orbit can come from dissipative processes that take place either in the star or in the planet. The circularization timescale due to processes in the planet is usually written (e.g., Mardling 2007) as

$$
\tau_{c i r c, p}=\frac{2}{21 n_{p}}\left(\frac{Q_{p}}{k_{p}}\right) q\left(\frac{a_{p}}{r_{p}}\right)^{5},
$$

where $Q_{p}$ is the planetary dissipation parameter, $k_{p}$ is the planetary tidal Love number (Goldreich \& Soter 1966) and $r_{p}$ is the planetary radius (See Mardling 2007 for an essential discussion of the slightly different definitions of the $\mathrm{Q}$ parameters by the different studies). For the corresponding timescale due to processes in the star, $\tau_{\text {circ,* }}$, one should replace $Q_{p}, k_{p}, r_{p}$ by the corresponding values of the star, $Q_{*}, k_{*}, R_{*}$, and switch between $m_{p}$ and $M_{*}$ (e.g., Carone \& Pätzold 2007). Unfortunately, as was already noted in the previous subsection, the theoretical estimate of the ratios $Q_{p} / k_{p}$ or $Q_{*} / k_{*}$ varies over one or two orders of magnitude (e.g., Lin et al. 1996; Trilling et al. 1998; Trilling 2000). For example, Trilling (2000) used $Q_{*} \geq 10^{6}$ for main-sequence stars and $1.5 \times 10^{4}-1.5 \times 10^{4}$ for PMS stars. Therefore, the theory is in a 'safe' situation, in which it can not be confronted with the observations.

\subsubsection{Small eccentricity induced by a second planet}

In Section 7.2 we discussed the Mazeh \& Shaham (1979) effect, in which a third distant companion can induce eccentricity in the binary orbit, even for a shortperiod orbit for which the tidal circularization had reduced the binary eccentricity. It was emphasized that the Mazeh \& Shaham effect can work even if the binary eccentricity starts at exactly zero value. This effect can have an impact on the evolution of close binaries, as the injected binary eccentricity invokes frictional forces inside the two stars that dissipate rotational energy and transfer angular momentum between the stellar rotation and the binary orbital motion.

Similar ideas have been proposed with regard to extrasolar planets. It was suggested that another, as-of-yet undetected planet pumps small eccentricity into the orbit of some known short-period planets. One such example is HD 209458b 
(Mazeh et al. 2000), the first transiting planet (Charbonneau et al. 2000; Henry 2000) that was found to have a somewhat surprisingly large radius (e.g., Burrows et al. 2000). The conjecture was that a small eccentricity of HD 209458b orbit (e.g., Laughlin et al. 2005) causes tidal dissipation inside the planet, which serves as another source of energy that inflates the planet (e.g., Bodenheimer et al. 2001; Bedenheimer et al. 2003; Gu et al. 2004; Mardling 2007). Two other examples are WASP-1b (Cameron et al. 2007) and HAT-P-1b (Bakos et al. 2007b).

The idea of a planetary eccentricity pumped by another as-of-yet undetected planet can be verified or refuted by further observations. Additional accurate radial-velocity observations can constrain the orbital eccentricity of the known short-period planets as well as detect unknown additional planets. Another avenue for accurate derivation of orbital eccentricity of the transiting planets is to detect the secondary eclipse, an approach recently carried out in the IR by the Spitzer satellite (e.g., Deming et al. 2005). When the orbital parameters of those planets are determined, we will be able to assess the validity of the idea of the pumping eccentricity, particularly for transiting planets for which we can determine the radius and eccentricity.

\subsection{Synchronization of planetary rotation}

Rotational synchronization of stars with short-period planets was not considered by the astrophysical community, as it was assumed that the masses of the planets are too small to synchronize the stellar rotation with the planetary orbital frequency. See, for example, the discussion of the stopping mechanism (Section 8.1.1), where we reviewed two cases with stellar rotational periods much longer than the orbital periods. At the same time, it was taken for granted that tidal dissipation inside the short-period planets has synchronized their own rotation with their orbital frequency (e.g., Rasio et al. 1996; Ogilvie \& Lin 2004).

Lubow et al. (1997) claimed that even a planet with a period of 200 days should be synchronized in 10 Gyrs (see also Ivanov \& Papaloizou 2007 and Ogilvie \& Lin 2007). As is commonly known, we find many planets with eccentric orbits at that period (see, for example, Figure 7). Therefore, we expect to have planets with orbital periods shorter than 200 days with pseudo-synchronized rotational periods (see detailed derivation of the pseudo-synchronization period by Ivanov \& Papaloizou 2007).

How can we observe planetary synchronization or pseudo-synchronization? Planets that are rotationally locked in a circular orbit have one hemisphere exposed to intense stellar insolation all the time, causing a significant difference in temperature between their day and night sides. The infrared emission of the day side was already detected by Spitzer through the secondary eclipse of HD 209458b (Deming et al. 2005; 2007), TrES-1 (Charbonneau et al. 2005), 189733b (Deming et al. 2006), and Gliese 436b (Deming et al. 2007; Demory et al. 2007), and even by the phase modulation of the non-transiting planet around $v$ And (Harrington et al. 2006). The exact periodicity and the phase of the IR modulation can confirm the synchronization assumption (e.g., Seager et al. 2005). Furthermore, IR 
modulation can reveal a signature of pseudo-synchronized planetary rotation.

As-of-yet, Spitzer detected IR modulations only of planets with circular or almost circular orbits with short periods, of the order of 3 days. Very recently the transit of a rather long-period eccentric planet, HD 17156b, has been discovered (Barbieri et al. 2007). It would be quite interesting to follow the IR modulation of this planet and find out if the planetary rotation is pseudo-synchronized with the orbital period of 21.2 days and with an eccentricity of 0.67 .

\subsection{Alignment of planetary motion with stellar rotation}

The a priori approach to extrasolar planetary alignment was similar to the early attitude to planetary eccentricity. Based on the solar system features, it was expected that the planetary orbital plane of motion would be aligned with the stellar rotational axis. The theory predicted that the dissipative processes in the protoplanetary disk tend to damp the initial inclinations of the planetesimals (e.g., Ward \& Hahn 1994; see also a later study by Cresswell et al. 2007). However, in the spirit of the new approach to the formation of planetary eccentricities by the interaction with the disk, Thommes \& Lissauer (2003), for example, suggested that when more than one planet is present in the system, the interaction with the material of the disk and the resonant interaction between the planets can turn the system noncoplanar. Interestingly, spin-orbit misalignment might cause tidal heating of the planet and contribute to its inflated radius, as was suggested by Winn \& Holman (2005) when trying to account for the large radius of HD 209458b (see Section 8.2.1). However, this idea was challenged by Levrard et al. (2007) and Fabrycky et al. (2007).

To observe the relative inclination of the planetary spin axis, we can use the RM effect for transiting planets (see Gaudi \& Winn 2007 for a thorough discussion of the effect and Section 6.1 for a discussion of the same effect observed in eclipsing binaries). The amplitude of the radial-velocity modulation of the RM effect is

$$
\Delta_{R M} \sim V_{*} \sin i_{*}\left(\frac{r_{p}}{R_{*}}\right)^{2},
$$

where $V_{*} \sin i_{*}$ is the rotational broadening of the stellar spectral lines. The RM amplitude of transiting planets is necessarily small, as $r_{p} / R_{*} \sim 0.1$. It can be as small as $\sim 10 \mathrm{~m} \mathrm{~s}^{-1}$, if $V_{*} \sin i_{*}$ is $1 \mathrm{~km} \mathrm{~s}^{-1}$. Nevertheless, the effect can have an amplitude as large as $\sim 50 \mathrm{~m} \mathrm{~s}^{-1}$, if $V_{*} \sin i_{*}$ is $5 \mathrm{~km} \mathrm{~s}^{-1}$. Such amplitude can be easily observed with the typical precision achieved for the study of extrasolar planets.

Recent observations (e.g., Queloz et al. 2000; Winn et al. 2006b; Narita 2007) that aimed specifically to follow the RM effect in a few transiting planets could not find any evidence for spin-orbit misalignment. This is true even for $147506 \mathrm{~b}$ (HATP-2b; Winn et al. 2007; Loeillet et al. 2007) for which a substantial eccentricity has been detected (Bakos et al. 2007a). Unfortunately, as discussed above, the theoretical interpretation of these findings is not clear. It could be attributed to the formation processes - planets are formed in aligned orbits, or it could be the 
outcome of some tidal dissipation between the spinning star and the planetary motion. We are still awaiting the discoveries of misaligned planets, which will help to clarify the broad scope of planetary alignment.

\section{In the era of large-scaled photometric surveys}

The advent of sensitive, large CCDs in the service of the astronomical community in the last decade or so has made large-scaled photometric surveys possible, yielding hundreds of accurate photometric measurements of many thousands of stars. Although the drive for these surveys was not always the study of binary stars, and eclipsing binaries in particular (e.g., MACHO, see Alcock et al. 1977), hundreds of eclipsing binaries have been discovered as a result of these surveys (e.g., Devor 2005).

In the past, even when a star was discovered to be an eclipsing binary, a special, additional observational effort was needed in order to acquire its lightcurve in order to derive its orbital parameters. In contrast, for eclipsing binaries discovered by large surveys we get, without any additional observational effort, lightcurves of unprecedented precision that are spread over a long period of time. Therefore, the present large-scaled photometric surveys are revolutionizing the study of eclipsing binaries, yielding new tools for the study of tidal interaction in particular. This section reviews two studies that have used two large surveys, MACHO and OGLE, which performed an intensive monitoring of the LMC for a few years. Both MACHO (Alcock et al. 1997; Derekas et al. 2007)) and OGLE (Udalski et al. 1998; Wyrzykowski et al. 2004) data sets were used to identify the largest sample of eclipsing binaries ever studied, with direct implications for the study of their tidal interaction. The goal of this section is to demonstrate the far-reaching potential of these surveys.

\subsection{Apsidal motion of eccentric binaries in the $L M C$}

Michalska \& Pigulski (2005) studied the discovered binaries in the LMC by the OGLE survey, and searched for binaries that are suitable for distance determination. Serendipitously, they found 14 binaries with significant apsidal precession. The LMC data spanned 2000 days, and therefore they could detect slow variation of the longitude of the periastron and derive long apsidal periods, of the order of thousands of years. In a follow-up paper, Michalska (2007) found evidence for apsidal motion in the data of an additional 11 binaries.

Unfortunately, the radii and masses of the components of the binaries of Michalska \& Pigulski and Michalska are not known, and therefore no comparison between the theory of apsidal motion induced by the tidal interaction and the observations is possible. However, we can try to take advantage of the fact that for the OGLE LMC data, most stars are in a limited magnitude range, and therefore their radii and masses are not very different from one another. Therefore, the apsidal precession period should depend mostly on the binary period. To check this hypothesis 


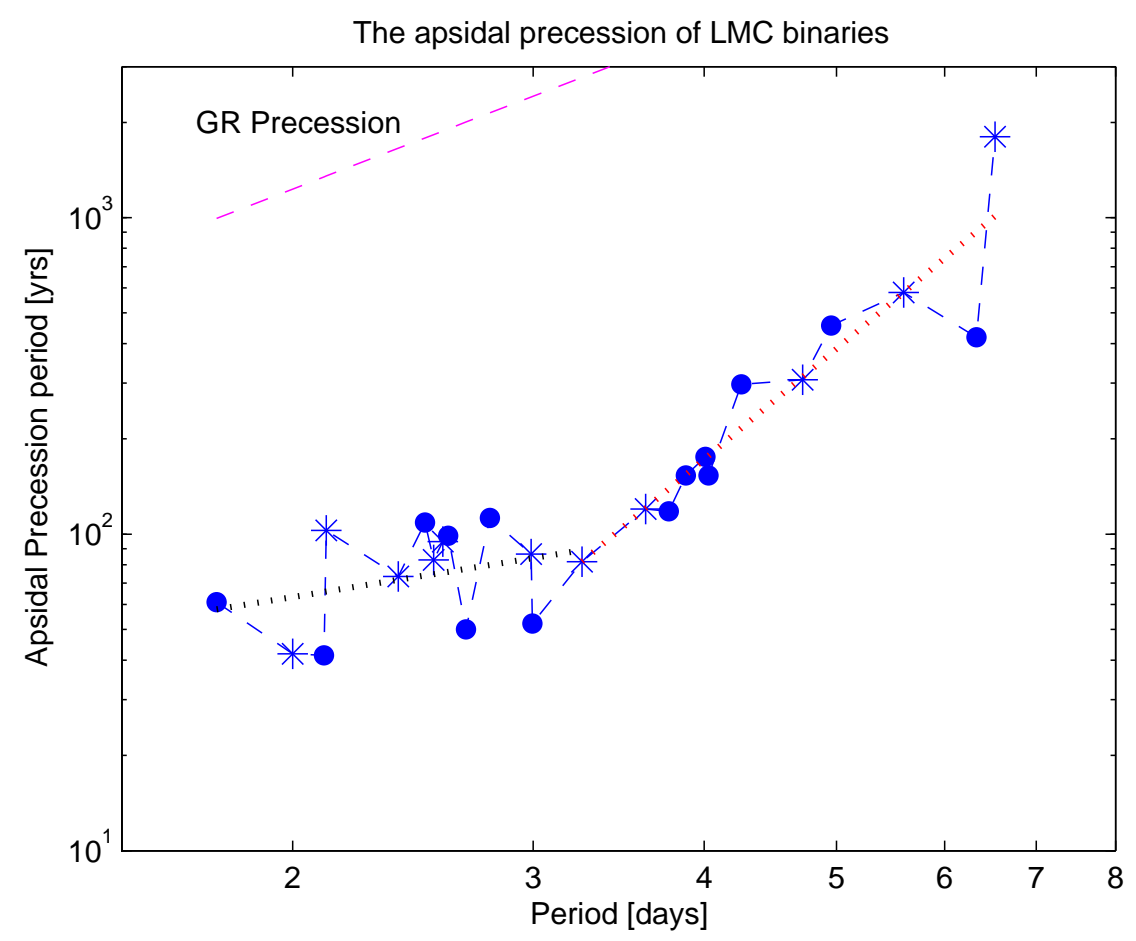

Fig. 8. The apsidal precession period as a function of the binary period for the LMC binaries. Binaries discussed by Michalska \& Pigulski (2005) are denoted by filled circles, and those discussed by Michalska (2007) by *. A dashed line connects the binaries sorted by period. Two straight lines were fitted, one for the first 14 short-period binaries, up to a period of 3.2 days, and the other for the binaries with longer periods. The expected GR precession is also plotted for binaries with total masses of $10 M_{\odot}$ and for almost circular orbits.

we plotted in Figure 8 the derived apsidal period as a function of the binary period. The period range is between about 2 and 7 days, and the apsidal period range is between 50 and 2000 years. For the binaries with periods below 3 days, the apsidal period shows a linear dependence on the binary period, as if the ratio $R_{1} / a$ in Eq. (3.1) is constant, where $R_{1}$ is the radius of the primary and $a$ is the semi-major axis. For the binaries with periods longer than 3 days the apsidal period is proportional to $P^{11 / 3}$, where $P$ is the binary period. Within the errors this slope is consistent with the exponent of Equation (3.4).

We note that one system, with a period of 6.33 days, has a substantially shorter precession period than the straight-line fit. This is OGLE LMC 05065201-6825466, the brightest star in the sample of 98 binaries analysed by Michalska \& Pigulski. This star, with a brightness of $V=14.04$, probably has a substantially larger 
radius than the other stars in Michalska \& Pigulski and Michalska samples, and therefore might have a shorter apsidal period.

The Michalska \& Pigulski and Michalska studies comprise the first systematic attempt to consider the apsidal motion in binaries detected by large-scaled systematic photometric surveys. We anticipate many more similar studies in the future.

\subsection{Circularization of eclipsing binaries of the SMC and LMC}

A seminal work that used the new sets of binaries to study tidal interaction was the study of North \& Zahn (2003), which analysed the detached eclipsing binaries cataloged by Udalski et al. (1998) in the SMC and by Alcock et al. (1997) in the LMC. Because of the magnitude range of the two surveys, all the binaries in these two samples have early-type primaries, mostly B-type stars (North \& Zahn 2003). Following Giuricin et al. (1984; see a review of their work in Section 4.3), North \& Zahn plotted the orbital eccentricity as a function of the fractional radii of the eclipsing binaries in the SMC and LMC. Like Giuricin et al. they tried to find out the cutoff fractional radius, above which all binaries are circularized. They were also trying to find out whether there is any difference between the circularization processes in early-type stars in our Galaxy and those in action in binaries in the SMC and LMC.

North \& Zahn pointed out that with the precision of the two surveys it was difficult to derive the orbital eccentricity from the lightcurve of an eclipsing binary. The phase of the secondary eclipse, which could be accurately determined, yielded only $e \cos \omega$, where $e$ is the eccentricity and $\omega$ is the longitude of the periastron. North \& Zahn overcame this problem by plotting the absolute value of $e \cos \omega$ instead of the eccentricity itself. As they were interested only in the statistical features of the sample, and in the cutoff fractional radius in particular, the fact that only $e \cos \omega$ was available to the analysis did not hamper their study.

North \& Zahn faced another problem. The given precision of the lightcurves of the two surveys did not really allow them to derive the radii of the two components of each binary. One could only get the sum of the two radii (in terms of the orbital separation), while the ratio of the two radii was not well determined. North \& Zahn had therefore to assume that the two stars have equal radii. Only with the aid of this assumption could they estimate the primary radius, which is, in fact, the average of the two radii. With these two modifications, the analysis of North \& Zahn (2003) yielded results similar to Giuricin et al. (1984). The cutoff fractional radius was about 0.25 , with no statistical difference between the early-type binaries of the Galaxy and those of SMC and LMC.

In a follow-up paper, North \& Zahn (2004) analysed many additional eclipsingbinary lightcurves, so that their samples included 148 binaries in the SMC and 353 binaries in the LMC. They then found, again, that the critical fractional radius was in the range $0.24-0.26$, irrespective of mass, surface gravity and metallicity. This value of the critical radius was consistent with the previous studies, and is compatible with the theory of Zahn (1975). 
North \& Zahn $(2003 ; 2004)$ did not use all the binaries detected in the OGLE LMC data. In fact, Wyrzykowski et al. (2004) identified 2580 eclipsing binaries in the LMC OGLE II photometric data alone. However, it is impractical to manually analyse such a large number of binaries. One would need an automated analysis to handle such a large set of binaries. Such algorithms were indeed constructed recently (e.g., Wyithe \& Wilson 2001; Devor \& Charbonneau 2006). Although the automated codes can err in some cases, for most of the binaries the algorithms yield the right solutions, rendering them powerful tools in the study of the statistical features of the close binary population (e.g., Mazeh et al. 2006).

Following this trend, Tamuz et al. (2006) constructed an automated algorithm, EBAS, to analyze eclipsing binary lightcurves, an algorithm which is based on the EBOP code (Popper \& Etzel 1981; Etzel 1981). The algorithm was then used by Mazeh et al. (2006) to analyze most of the eclipsing binaries detected by Wyrzykowski et al. (2004) in the OGLE LMC data. Mazeh et al. then plotted the eccentricity as a function of the fractional radius, with two minor changes relative to the plot of North \& Zahn. First, instead of the primary fractional radius, they plotted the total fractional radii $R_{\text {total }}=R_{1}+R_{2}$ - where $R_{1}$ and $R_{2}$ are the fractional radii of the primary and secondary, respectively. Second, they specifically plotted $e \cos \omega$ instead of its absolute value. They found that the critical total fractional radius is 0.5 , consistent with the North \& Zahn result.

Here we follow Mazeh et al. (2006) and plot in Figure $9 e \cos \omega$ as a function of the total fractional radius for all 1335 binaries with $19 \leq I \leq 17$ analysed by Tamuz et al. (2005). We also plotted a straight line upper envelope, that goes like

$$
\pm e \cos \omega=0.857-1.714 R_{\text {total }},
$$

which implies, if we ignore the $\cos \omega$ factor, that the periastron distance, $1-e$, in terms of the semi-major axis, is

$$
2\left(R_{1}+R_{2}\right) \lesssim 1-e
$$

(Mazeh et al. 2006). This could mean that if the two stars got too close at periastron, to the extent that the distance between them was less than twice their total radius, then circularization processes went into action and the eccentricity was reduced until the two stars became further apart. This is not the only interpretation. One can imagine that binaries were not formed too close together, lest they violate this relation. Obviously, the present distribution could be the caused by the two scenarios - formation and tidal evolution.

Note that similarly to Giuricin et al. (see Section 4.3), we also find a few binaries with a primary radius larger than 0.3 which display small but significant eccentricities. We also tend to attribute these eccentricities to unseen third distant companions, an effect suggested by Mazeh \& Shaham (1979; see detailed discussion in Section 7.2).

The North \& Zahn $(2003 ; 2004)$ and Mazeh et al. (2006) studies were the first to use large-scaled photometric surveys to touch upon circularization interaction. We anticipate many more, similar studies in the future. 




Fig. 9. The eccentricity, multiplied by $\cos \omega$, of the OGLE eclipsing binaries of the LMC as a function of total fractional radius. Included are 1335 binaries with $17 \leq I \leq 19$. The dashed line represents the upper envelope (see text).

\section{Discussion}

This paper has attempted to review a large corpus of observational evidence for tidal interaction in close binary systems, including short-period extrasolar planets. We first discussed two tidal effects that can be observed directly - the ellipsoidal variation and the apsidal motion. Unfortunately, these two effects have been detected in the past in only a small number of stars, because the detection required special photometric observations which involved a considerable effort. The small number of known binaries with detected ellipsoidal modulation or apsidal motion allowed for only a limited confrontation between the theory and the observations. To perform a comprehensive confrontation we need many more binaries with accurate measurements of these two effects.

Ellipsoidal modulation was mostly used in the past to study known spectroscopic binaries (e.g., HD 149420 - Fekel \& Henry 2006), or binaries with compact objects. This situation is being changed dramatically, as ground-based large-scaled photometric surveys have been operated in the last decade. We already discussed 
two such 1-m class exhaustive projects, MACHO (Alcock et al. 1993) and OGLE (e.g., Udalski et al. 1997). Furthermore, a new wave of observational effort to search for transiting planets by small, 10-cm class, telescopes is now at its full thrust (e.g., HATnet - Bakos et al. 2004; XO - McCullough et al. 2005; WASP - Pollacco et al. 2006; TrES - Alonso et al. 2007). These projects are collecting an unprecedented number of photometric datasets that harbor thousands of ellipsoidal binaries.

The amplitude of the ellipsoidal modulation can be of the order of a few percent, and therefore should be easily detected in these large photometric datasets. Detecting ellipsoidal variables can become a tool for discovering new binaries. In such cases, one must distinguish between the ellipsoidal modulation and other possible variations, particularly stellar spots and pulsation. For that purpose, one must rely on the theoretical shape of the modulation and on observations in different passbands. A first step towards this goal has been performed already by Derekas et al. (2006), who identified ellipsoidal variables in the sample of variable pulsating red giants within the MACHO data.

The huge datasets are having another effect on the study of close binaries: they are already revealing a large number of new eclipsing binaries (e.g., Devor 2005). Therefore the number of known eclipsing binaries will increase in the next few years by one or two orders of magnitude. The tens of thousands of new eclipsing binaries will revolutionize our understanding of close binaries in general and their statistical features in particular. These datasets will reveal many other effects of eclipsing binaries, such as apsidal motion and third distant companions through the time-travel effect. We will then be well equipped to confront the theory of apsidal motion with observations, and learn more about the frequency and distribution of triple systems. We will be able to verify conjectures regarding the correlation between short-period binaries and distant companions.

The influx of photometric data is about to increase, as more automated photometric surveys with 1-m class telescopes will set into action. This includes SkyMaper (Bayliss \& Sackett 2007), Las Cumbres Observatory Global Telescope (Brown et al. 2007), Pan-STARRS (e.g., Holman et al. 2007), and finally LSST (e.g., Strauss et al. 2006). One such binary was discovered already by Blake et al. (2007), using the Sloan Digital Sky Survey II. This influx of photometric data will be complemented by extremely accurate datasets from space missions, which will have an immediate impact on binary studies. This trend has already been started by HIPPARCOS (e.g., Jorissen et al. 2004), and will be joined by CoRoT (Maceroni and Ribas 2007), Kepler (Koch et al. 2007) and GAIA (e.g., Niarchos 2006) missions. For the future of binary studies in the 'brave new era,' see a review by Guinan \& Engle (2006).

We have discussed the long-term tidal processes of circularization, synchronization and spin alignment. As was reviewed, binary circularization has attracted many studies, particularly of the transition period between circular and eccentric binaries. Nevertheless, no consensus has been reached with regard to the theoretical timescale of the tidal processes, and even the dominant dissipation process behind the circularization is still being debated. Synchronization and alignment were 
studied much less. For example, the number of eclipsing binaries with observed $\mathrm{RM}$ effect by which we study the spin-orbit alignment is only six, an extremely small number. Modern spectrographs with efficient digital detectors can observe many more eclipsing binaries, and the development of tools to analyze the observations can enable us to accurately derive the spin-orbit inclination. The results may well be crucial for our understanding of binary formation, as this will help us estimate the primordial alignment of binary population. In case binaries are formed with some spread of spin-orbit inclinations, we should also expect coeval samples of binaries to exhibit a transition period between aligned and non-aligned binaries. Such an observed transition can serve as another confirmation of the theory of tidal interaction. It would be of extreme importance to compare the aligned/non-aligned transition period with the pseudo-synchronized/non-pseudosynchronized and circularized/eccentric transition periods.

An additional feature of the new large accurate photometric datasets is their long time span, of the order of a few years, that will enable us to detect minute variations which disclose long-term variations. The search of Michalska \& Pigulski (2005) and Michalska (2007) for evidence of apsidal motion discussed above is one example. The works of Kubiak et al. (2006) and Pilecki et al. (2007) are two other examples of studies that utilized the long time span of the new data sets. Some of the yet-to-come studies will be able to find eventually direct evidence for long-term tidal processes in close binaries.

Finally, we have discussed in brief some ideas about tidal evolution of shortperiod extrasolar planets. Many of the short-period planets are being discovered by photometry which detects how these planets eclipse their parent stars. The transiting planets hold a higher potential for the study of tidal effects, as we know so much more about them, including their masses and radii. They can be used as clean and unique laboratories to study tidal effects, as the complexity of their systems is reduced because of the small masses and radii of the planets. As of October 2007, 27 transiting planets are known, which is about half of the short-period extrasolar planets, the others have been discovered by radial-velocity measurements. We anticipate that the balance between the radial-velocity and transiting planets will change in the near future in favour of the transiting planets. The rapidly increasing number of transiting planets will enable us to study the possible correlation between presumed tidal effects and the stopping mechanism, the inflated radii of some planets, synchronization of the stars and planets and their spin-orbit alignment. A comprehension of these features will help us assess the role of tidal interaction in the orbital evolution of short-period extrasolar planets.

\section{Acknowledgements}

Special thanks to Jean-Paul Zahn for inviting me to the Oleron summer school to give a series of lectures on the subject of this review. This paper emerged from those lectures. I am deeply thankful for his support and patience during the long period of writing the manuscript and for his enlightening comments. I am indebted to Soeren Meibom and Itzhak Goldman for many fruitful discussions and 
enlightening comments. Special thanks to Peter Eggleton, Dan Fabrycky, Scott Gaudi, Mike Lecar, Doug Lin, Andrei Tokovinin and Guillermo Torres who read an early version of the manuscript and send me thoughtful comments and suggestions. I wish to heartily thank my students Avi Shporer, Aviv Ofir and Yevgeny Tsodikovich for technical help with handling the data and with the plotting.

This work was supported by the Israeli Science Foundation through grant no. 03/233. I thank the Swiss National Science Foundation and the Smithsonian Institution for supporting my stay at the Geneva Observatoire and at the CfA, during which I worked on this paper.

\section{References}

Abt, H. A. and Boonyarak, C.: 2004, ApJ 616, 562

Abt, H. A., Levato, H., and Grosso, M.: 2002, ApJ 573, 359

Aerts, C., Handler, G., Arentoft, T., Vandenbussche, B., Medupe, R., and Sterken, C.: 2002, MNRAS 333, L35

Albrecht, S., Reffert, S., Snellen, I., Quirrenbach, A., and Mitchell, D. S.: 2007, $A ध A$ 474, 565

Alcock, C., Allsman, R. A., Alves, D., Axelrod, T. S., Becker, A. C., Bennett, D. P., Cook, K. H., Freeman, K. C., Griest, K., Lacy, C. H. S., Lehner, M. J., Marshall, S. L., Minniti, D., Peterson, B. A., Pratt, M. R., Quinn, P. J., Rodgers, A. W., Stubbs, C. W., Sutherland, W., and Welch, D. L.: 1997, AJ $\mathbf{1 1 4}, 326$

Alcock, C., Allsman, R. A., Axelrod, T. S., Bennett, D. P., Cook, K. H., Park, H. S., Marshall, S. L., Stubbs, C. W., Griest, K., Perlmutter, S., Sutherland, W., Freeman, K. C., Peterson, B. A., Quinn, P. J., and Rodgers, A. W.: 1993, in B. T. Soifer (ed.), Sky Surveys. Protostars to Protogalaxies, Vol. 43 of Astron. Soc. Pacific Conf. Ser., p. 291

Alonso, R., Brown, T. M., Charbonneau, D., Dunham, E. W., Belmonte, J. A., Deeg, H. J., Fernández, J. M., Latham, D. W., Mandushev, G., O’Donovan, F. T., Rabus, M., and Torres, G.: 2007, in C. Afonso, D. Weldrake, and T. Henning (eds.), Transiting Extrapolar Planets Workshop, Vol. 366 of Astron. Soc. Pacific Conf. Ser., p. 13

Andersen, J., Nordstrom, B., Garcia, J. M., and Giménez, A.: 1987, A\& A 174, 107

Ash, T. D. C., Reynolds, A. P., Roche, P., Norton, A. J., Still, M. D., and MoralesRueda, L.: 1999, MNRAS 307, 357

Badalian, H. S. and Erastova, L. K.: 1970, Astronomicheskij Tsirkulyar 591, 4

Bahcall, J. N., Pinsonneault, M. H., and Basu, S.: 2001, ApJ 555, 990

Bahcall, J. N. and Ulrich, R. K.: 1988, Reviews of Modern Physics 60, 297

Bakos, G., Noyes, R. W., Kovács, G., Stanek, K. Z., Sasselov, D. D., and Domsa, I.: 2004, PASP 116, 266

Bakos, G. A., Kovacs, G., Torres, G., Fischer, D. A., Latham, D. W., Noyes, R. W., Sasselov, D. D., Mazeh, T., Shporer, A., Butler, R. P., Stefanik, R. P., 
Fernandez, J. M., Sozzetti, A., Pal, A., Johnson, J., Marcy, G. W., Winn, J., Sipocz, B., Lazar, J., Papp, I., and Sari, P.: 2007a, ApJ 670, 826

Bakos, G. Á., Noyes, R. W., Kovács, G., Latham, D. W., Sasselov, D. D., Torres, G., Fischer, D. A., Stefanik, R. P., Sato, B., Johnson, J. A., Pál, A., Marcy, G. W., Butler, R. P., Esquerdo, G. A., Stanek, K. Z., Lázár, J., Papp, I., Sári, P., and Sipőcz, B.: 2007b, ApJ 656, 552

Baliunas, S. L., Donahue, R. A., Soon, W. H., Horne, J. H., Frazer, J., WoodardEklund, L., Bradford, M., Rao, L. M., Wilson, O. C., Zhang, Q., Bennett, W., Briggs, J., Carroll, S. M., Duncan, D. K., Figueroa, D., Lanning, H. H., Misch, T., Mueller, J., Noyes, R. W., Poppe, D., Porter, A. C., Robinson, C. R., Russell, J., Shelton, J. C., Soyumer, T., Vaughan, A. H., and Whitney, J. H.: 1995, ApJ 438, 269

Barbieri, M., Alonso, R., Laughlin, G., Almenara, J. M., Bissinger, R., Davies, D., Gasparri, D., Guido, E., Lopresti, C., Manzini, F., and Sostero, G.: 2007, astro-ph/0710.0898

Bate, M. R., Bonnell, I. A., and Bromm, V.: 2002, MNRAS 336, 705

Batten, A. H., Fletcher, J. M., and Mann, P. J.: 1978, Publ. Dominion Astrophys. Obs. Victoria 15, 121

Batten, A. H., Morbey, C. L., Fekel, F. C., and Tomkin, J.: 1979, PASP 91, 304

Bayliss, D. D. R. and Sackett, P. D.: 2007, in C. Afonso, D. Weldrake, and T. Henning (eds.), Transiting Extrapolar Planets Workshop, Vol. 366 of Astron. Soc. Pacific Conf. Ser., p. 320

Beatty, T. G., Fernández, J. M., Latham, D. W., Bakos, G. Á., Kovács, G., Noyes, R. W., Stefanik, R. P., Torres, G., Everett, M. E., and Hergenrother, C. W.: 2007, ApJ 663, 573

Benvenuto, O. G., Serenelli, A. M., Althaus, L. G., Barbá, R. H., and Morrell, N. I.: $2002, M N R A S \mathbf{3 3 0}, 435$

Beust, H.: 2003, A\&SA 400, 1129

Beust, H., Corporon, P., Siess, L., Forestini, M., and Lagrange, A.-M.: 1997, A\&BA 320, 478

Bildsten, L., Chakrabarty, D., Chiu, J., Finger, M. H., Koh, D. T., Nelson, R. W., Prince, T. A., Rubin, B. C., Scott, D. M., Stollberg, M., Vaughan, B. A., Wilson, C. A., and Wilson, R. B.: 1997, ApJS 113, 367

Blake, C. H., Torres, G., Bloom, J. S., and Gaudi, B. S.: 2007, astro-ph/0707.3604

Bodenheimer, P., Laughlin, G., and Lin, D. N. C.: 2003, ApJ 592, 555

Bodenheimer, P., Lin, D. N. C., and Mardling, R. A.: 2001, ApJ 548, 466

Bodenheimer, P. and Pollack, J. B.: 1986, Icarus 67, 391

Bonfils, X., Mayor, M., Delfosse, X., Forveille, T., Gillon, M., Perrier, C., Udry, S., Bouchy, F., Lovis, C., Pepe, F., Queloz, D., Santos, N. C., and Bertaux, J.-L.: 2007, A\&A 474, 293

Bonnell, I. A. and Bate, M. R.: 2005, MNRAS 362, 915

Borkovits, T., Forgács-Dajka, E., and Regály, Z.: 2004, A $\& A$ 426, 951

Borkovits, T., Forgács-Dajka, E., and Regály, Z.: 2005, in A. Claret, A. Giménez, and J.-P. Zahn (eds.), Tidal Evolution and Oscillations in Binary Stars, Vol. 333 of Astron. Soc. Pacific Conf. Ser., p. 128 
Borkovits, T., Forgács-Dajka, E., and Regály, Z.: 2007, A\& A 473, 191

Boss, A. P.: 1997, Science 276, 1836

Bozkurt, Z. and Değirmenci, Ö. L.: 2007, MNRAS 379, 370

Brown, E. W.: 1936, MNRAS 97, 56

Brown, E. W.: 1937, MNRAS 97, 388

Brown, T., Rosing, W. E., Baliber, N., Hidas, M., and Street, R.: 2007, in American Astron. Soc. Meeting Abstracts, Vol. 210, p. 66.01

Bulut, I. and Demircan, O.: 2007, MNRAS 378, 179

Burkert, A. and Ida, S.: 2007, ApJ 660, 845

Burrows, A., Guillot, T., Hubbard, W. B., Marley, M. S., Saumon, D., Lunine, J. I., and Sudarsky, D.: 2000, ApJL 534, L97

Butler, R. P., Vogt, S. S., Marcy, G. W., Fischer, D. A., Wright, J. T., Henry, G. W., Laughlin, G., and Lissauer, J. J.: 2004, ApJ 617, 580

Cameron, A. C., Bouchy, F., Hébrard, G., Maxted, P., Pollacco, D., Pont, F., Skillen, I., Smalley, B., Street, R. A., West, R. G., Wilson, D. M., Aigrain, S., Christian, D. J., Clarkson, W. I., Enoch, B., Evans, A., Fitzsimmons, A., Fleenor, M., Gillon, M., Haswell, C. A., Hebb, L., Hellier, C., Hodgkin, S. T., Horne, K., Irwin, J., Kane, S. R., Keenan, F. P., Loeillet, B., Lister, T. A., Mayor, M., Moutou, C., Norton, A. J., Osborne, J., Parley, N., Queloz, D., Ryans, R., Triaud, A. H. M. J., Udry, S., and Wheatley, P. J.: 2007, MNRAS $\mathbf{3 7 5}, 951$

Cardini, D. and Cassatella, A.: 2007, ApJ 666, 393

Carone, L. and Pätzold, M.: 2007, Planet. Space Sci. 55, 643

Cester, B., Fedel, B., Giuricin, G., Mardirossian, F., Mezzetti, M., and Predolin, F.: 1979, Mem. Soc. Astron. Italiana 50, 553

Charbonneau, D., Allen, L. E., Megeath, S. T., Torres, G., Alonso, R., Brown, T. M., Gilliland, R. L., Latham, D. W., Mandushev, G., O'Donovan, F. T., and Sozzetti, A.: 2005, ApJ 626, 523

Charbonneau, D., Brown, T. M., Latham, D. W., and Mayor, M.: 2000, ApJL $\mathbf{5 2 9}$, L45

Charles, P. A. and Coe, M. J.: 2006, Optical, ultraviolet and infrared observations of X-ray binaries, p. 215, Compact stellar X-ray sources

Chevalier, R. A.: 1975, ApJ 199, 556

Chiang, E. I. and Murray-Clay, R. A.: 2004, ApJ 607, 913

Choi, P. I. and Herbst, W.: 1996, AJ 111, 283

Claret, A.: 1998, A\&A 330, 533

Claret, A.: 1999, $A \& \mathcal{G}$ 350, 56

Claret, A.: 2007, $A \mathscr{B} A$ 467, 1389

Claret, A. and Giménez, A.: 1993, A\&A 277, 487

Claret, A. and Willems, B.: 2002, A\&A 388, 518

Company, R., Portilla, M., and Giménez, A.: 1988, ApJ 335, 962

Covino, E., Melo, C., Alcalá, J. M., Torres, G., Fernández, M., Frasca, A., and Paladino, R.: 2001, $A \mathscr{E} A$ 375, 130

Cowling, T. G.: 1938, MNRAS 98, 734

Cresswell, P., Dirksen, G., Kley, W., and Nelson, R. P.: 2007, astro-ph/0707.2225 
Croll, B., Walker, G. A. H., Kuschnig, R., Matthews, J. M., Rowe, J. F., Walker, A., Rucinski, S. M., Hatzes, A. P., Cochran, W. D., Robb, R. M., Guenther, D. B., Moffat, A. F. J., Sasselov, D., and Weiss, W. W.: 2006, ApJ 648, 607

D'Angelo, C., van Kerkwijk, M. H., and Rucinski, S. M.: 2006, AJ 132, 650

Davidson, K. and Ostriker, J. P.: 1973, ApJ 179, 585

Deming, D., Harrington, J., Laughlin, G., Seager, S., Navarro, S. B., Bowman, W. C., and Horning, K.: 2007a, ApJL 667, L199

Deming, D., Harrington, J., Seager, S., and Richardson, L. J.: 2006, ApJ 644, 560

Deming, D., Richardson, L. J., and Harrington, J.: 2007b, MNRAS 378, 148

Deming, D., Seager, S., Richardson, L. J., and Harrington, J.: 2005, Nature 434, 740

Demory, B. ., Gillon, M., Barman, T., Bonfils, X., Mayor, M., Mazeh, T., Pont, F., Queloz, D., Udry, S., Bouchy, F., Delfosse, X., Forveille, T., Mallmann, F., Pepe, F., and Perrier, C.: 2007, astro-ph/0706.0224

Derekas, A., Kiss, L. L., and Bedding, T. R.: 2007, ApJ 663, 249

Derekas, A., Kiss, L. L., Bedding, T. R., Kjeldsen, H., Lah, P., and Szabó, G. M.: 2006, ApJL 650, L55

Devor, J.: 2005, ApJ 628, 411

Devor, J. and Charbonneau, D.: 2006, Ap\&SS 304, 351

Duquennoy, A. and Mayor, M.: 1991, A\&A 248, 485

Duquennoy, A., Mayor, M., Andersen, J., Carquillat, J. M., and North, P.: 1992, $A \& A$ 254, L13

Durisen, R. H., Sterzik, M. F., and Pickett, B. K.: 2001, A\& A 371, 952

Dziembowski, W. A. and Pamyatnykh, A. A.: 1991, A\&A 248, L11

Eaton, J. A. and Henry, G. W.: 2007, PASP 119, 259

Eggleton, P.: 2006, Evolutionary Processes in Binary and Multiple Stars, Cambridge University Press

Eggleton, P. P. and Kiseleva-Eggleton, L.: 2001, ApJ 562, 1012

Eggleton, P. P. and Kisseleva-Eggleton, L.: 2006, ApSS 304, 75

Etzel, P. B.: 1981, in E. B. Carling and Z. Kopal (eds.), Photometric and Spectroscopic Binary Systems, p. 111

Fabbiano, G. and Schreier, E. J.: 1977, ApJ 214, 235

Fabrika, S.: 2004, Astrophys. Space Phys. Reviews 12, 1

Fabrycky, D. and Tremaine, S.: 2007, astro-ph/0705.4285

Fabrycky, D. C., Johnson, E. T., and Goodman, J.: 2007, ApJ 665, 754

Fekel, F. C. and Henry, G. W.: 2006, AJ 131, 1724

Ford, E. B., Kozinsky, B., and Rasio, F. A.: 2000, ApJ 535, 385

Ford, E. B., Kozinsky, B., and Rasio, F. A.: 2004, ApJ 605, 966

Furtig, W. and Meinunger, L.: 1976, Inform. Bull. Variable Stars 1104, 1

Gaudi, B. S. and Winn, J. N.: 2007, ApJ 655, 550

Gelino, D. M., Balman, Ş., Kızıloğlu, Ü., Yılmaz, A., Kalemci, E., and Tomsick, J. A.: 2006, ApJ 642, 438

Georgakarakos, N.: 2002, MNRAS 337, 559

Georgakarakos, N.: 2004, Celestial Mechanics and Dynamical Astronomy 89, 63 
Giacconi, R., Gursky, H., Kellogg, E., Levinson, R., Schreier, E., and Tananbaum, H.: 1973, ApJ 184, 227

Gillon, M., Pont, F., Demory, B.-O., Mallmann, F., Mayor, M., Mazeh, T., Queloz, D., Shporer, A., Udry, S., and Vuissoz, C.: 2007, A\&3A 472, L13

Giménez, A.: 1990, in C. Ibanoglu (ed.), NATO ASIC Proc. 319: Active Close Binaries Proceedings, NATO Advanced Study Institute, p. 137

Giménez, A.: 2006, ApJ 650, 408

Giménez, A., Kim, C.-H., and Nha, I.-S.: 1987, MNRAS 224, 543

Giménez, A. and Margrave, T. E.: 1985, AJ 90, 358

Giuricin, G., Mardirossian, F., and Mezzetti, M.: 1984, A\&A 134, 365

Goldman, I. and Mazeh, T.: 1991, ApJ 376, 260

Goldman, I. and Mazeh, T.: 1994, ApJ 429, 362

Goldreich, P. and Nicholson, P. D.: 1989, ApJ 342, 1079

Goldreich, P. and Sari, R.: 2003, ApJ 585, 1024

Goldreich, P. and Soter, S.: 1966, Icarus 5, 375

Goldreich, P. and Tremaine, S.: 1980, ApJ 241, 425

Goodman, J. and Dickson, E. S.: 1998, ApJ 507, 938

Goodman, J. and Oh, S. P.: 1997, ApJ 486, 403

Gray, D. F.: 2005, The Observation and Analysis of Stellar Photospheres, Cambridge University Press

Griffin, R. F.: 1984, The Observatory 104, 148

Griffin, R. F. and Gunn, J. E.: 1978, AJ 83, 1114

Griffin, R. F. and Gunn, J. E.: 1981, AJ 86, 588

Gu, P.-G., Bodenheimer, P. H., and Lin, D. N. C.: 2004, ApJ 608, 1076

Guinan, E. F. and Engle, S. G.: 2006, Ap\&SS 304, 5

Guinan, E. F. and Maloney, F. P.: 1985, AJ 90, 1519

Guinan, E. F., Ribas, I., Fitzpatrick, E. L., Giménez, Á., Jordi, C., McCook, G. P., and Popper, D. M.: 2000, ApJ 544, 409

Haisch, Jr., K. E., Lada, E. A., and Lada, C. J.: 2001, ApJL 553, L153

Halbwachs, J. L., Mayor, M., and Udry, S.: 2005, A\& $A$ 431, 1129

Hamilton, C. M., Herbst, W., Mundt, R., Bailer-Jones, C. A. L., and Johns-Krull, C. M.: 2003, ApJL 591, L45

Hamilton, C. M., Herbst, W., Vrba, F. J., Ibrahimov, M. A., Mundt, R., BailerJones, C. A. L., Filippenko, A. V., Li, W., Béjar, V. J. S., Ábrahám, P., Kun, M., Moór, A., Benkő, J., Csizmadia, S., DePoy, D. L., Pogge, R. W., and Marshall, J. L.: 2005, $A J$ 130, 1896

Harrington, J., Hansen, B. M., Luszcz, S. H., Seager, S., Deming, D., Menou, K., Cho, J. Y.-K., and Richardson, L. J.: 2006, Science 314, 623

Harrington, R. S.: 1968, AJ 73, 190

Harrington, R. S.: 1969, Celestial Mechanics 1, 200

Harrison, T. E., Howell, S. B., Szkody, P., and Cordova, F. A.: 2007, AJ 133, 162

Henry, G. W., Marcy, G. W., Butler, R. P., and Vogt, S. S.: 2000, ApJL 529, L41

Herbst, W., Hamilton, C. M., Vrba, F. J., Ibrahimov, M. A., Bailer-Jones, C. A. L., Mundt, R., Lamm, M., Mazeh, T., Webster, Z. T., Haisch, K. E., Williams, E. C., Rhodes, A. H., Balonek, T. J., Scholz, A., and Riffeser, A.: 2002, PASP 
114, 1167

Herbst, W. and Moran, E. C.: 2005, ApJ 630, 400

Holman, M., Touma, J., and Tremaine, S.: 1997, Nature 386, 254

Holman, M. J., Protopapas, P., and Tholen, D. J.: 2007, in AAS/Division for

Planetary Sciences Meeting Abstracts, Vol. 39, p. 52.08

Hosokawa, Y.: 1953, PASJ 5, 88

Hube, D. P.: 1976, Informational Bulletin on Variable Stars 1123, 1

Hube, D. P. and Couch, J. S.: 1982, ApSS 81, 357

Hulse, R. A. and Taylor, J. H.: 1975, ApJL 195, L51

Hut, P.: 1981, A\&A 99, 126

Hut, P.: 1982a, A\&A 116, 351

Hut, P.: 1982b, $A \& A$ 110, 37

Isobe, S., Noguchi, M., Ohtsubo, J., Baba, N., Miura, N., Tanaka, T., and Ni-Ino, M.: 1992, Publ. National Astron. Obs. Japan 2, 459

Ivanov, P. B. and Papaloizou, J. C. B.: 2007, MNRAS 376, 682

Janssen, T. and van Kerkwijk, M. H.: 2005, A $\mathscr{E} A$ 439, 433

Jeffery, C. S.: 1984, MNRAS 207, 323

Jetsu, L., Pelt, J., and Tuominen, I.: 1999, A\&BA 351, 212

Jha, S., Torres, G., Stefanik, R. P., Latham, D. W., and Mazeh, T.: 2000, MNRAS $\mathbf{3 1 7}, 375$

Johnson, J. A., Marcy, G. W., Hamilton, C. M., Herbst, W., and Johns-Krull, C. M.: 2004, AJ 128, 1265

Jones, H. R. A., Butler, R. P., Tinney, C. G., Marcy, G. W., Carter, B. D., Penny, A. J., McCarthy, C., and Bailey, J.: 2006, MNRAS 369, 249

Jorissen, A., Jancart, S., and Pourbaix, D.: 2004, in R. W. Hilditch, H. Hensberge, and K. Pavlovski (eds.), Spectroscopically and Spatially Resolving the Components of the Close Binary Stars, Vol. 318 of Astron. Soc. Pacific Conf. Ser., pp 141-143

Juric, M. and Tremaine, S.: 2007, astro-ph/0703160

Katz, J. I.: 1973, Nature 246, 87

Kearns, K. E. and Herbst, W.: 1998, AJ 116, 261

Kiseleva, L. G., Eggleton, P. P., and Mikkola, S.: 1998, MNRAS 300, 292

Koch, D., Borucki, W., Basri, G., Brown, T., Caldwell, D., Christensen-Dalsgaard, J., Cochran, W., Devore, E., Dunham, E., Gautier, T. N., Geary, J., Gilliland, R., Gould, A., Jenkins, J., Kondo, Y., Latham, D., Lissauer, J., and Monet, D.: 2007, in IAU Symposium 240: Binary Stars as Critical Tools 85 Tests in Contemporary Astrophysics, pp 236-243

Koch, R. H. and Hrivnak, B. J.: 1981, AJ 86, 438

Konacki, M., Torres, G., Jha, S., and Sasselov, D. D.: 2003, Nature 421, 507

Königl, A.: 1991, ApJL 370, L39

Kopal, Z.: 1942, Proceedings of the National Academy of Science 28, 133

Kopal, Z.: 1959, Close binary systems, The International Astrophysics Series, London: Chapman \& Hall, 1959

Kozai, Y.: 1962, AJ 67, 591

Krymolowski, Y. and Mazeh, T.: 1999, MNRAS 304, 720 
Krzeminski, W.: 1974, Memorie della Societa Astronomica Italiana 45, 877

Kubiak, M., Udalski, A., and Szymanski, M. K.: 2006, Acta Astronomica 56, 253

Kuchner, M. J. and Lecar, M.: 2002, ApJL 574, L87

Latham, D., Mathieu, R. D., Milone, A. A. E., and Davis, R. J.: 1992a, in A. Duquennoy and M. Mayor (eds.), Binaries as Tracers of Stellar Formation. Proceedings of a Workshop held in Bettmeralp, Switzerland, Sept. 1991, in honor of Dr. Roger Griffin; Cambridge University Press, p. 132

Latham, D. W., Mathieu, R. D., Milone, A. A. E., and Davis, R. J.: 1992b, in Y. Kondo, R. Sistero, and R. S. Polidan (eds.), IAU Symp. 151: Evolutionary Processes in Interacting Binary Stars, p. 471

Latham, D. W., Stefanik, R. P., Mazeh, T., Mayor, M., and Burki, G.: 1989, Nature 339, 38

Latham, D. W., Stefanik, R. P., Torres, G., Davis, R. J., Mazeh, T., Carney, B. W., Laird, J. B., and Morse, J. A.: 2002, AJ 124, 1144

Laughlin, G., Marcy, G. W., Vogt, S. S., Fischer, D. A., and Butler, R. P.: 2005, ApJL 629, L121

Lecar, M., Wheeler, J. C., and McKee, C. F.: 1976, ApJ 205, 556

Levato, H., Malaroda, S., Morrell, N., and Solivella, G.: 1987, ApJS 64, 487

Levrard, B., Correia, A. C. M., Chabrier, G., Baraffe, I., Selsis, F., and Laskar, J.: 2007, $A \& A$ 462, L5

Lin, D. N. C., Bodenheimer, P., and Richardson, D. C.: 1996, Nature 380, 606

Lin, D. N. C. and Papaloizou, J.: 1986, ApJ 309, 846

Loeillet, B., Shporer, A., Bouchy, F., Pont, F., Mazeh, T., Beuzit, J. L., Boisse, I., Bonfils, X., Da Silva, R., Delfosse, X., Desort, M., Ecuvillon, A., Forveille, T., Galland, F., Gallenne, A., Hebrard, G., Lagrange, A. M., Lovis, C., Mayor, M., Moutou, C., Pepe, F., Perrier, C., Queloz, D., Segransan, D., Sivan, J. P., Santos, N. C., Tsodikovich, Y., Udry, S., and Vidal-Madjar, A.: 2007, astro$\mathrm{ph} / 0707.0679$

Lomb, N. R.: 1976, Ap\&SS 39, 447

Lubow, S. H., Tout, C. A., and Livio, M.: 1997, ApJ 484, 866

Lyytinen, J., Johansson, P., Jetsu, L., Esko, E., Hackman, T., Hall, D. S., Henry, G. W., Kontinen, S., Könönen, P., Maisala, S., Palviainen, A., and Ryynänen, K.: 2002, A\&A 383, 197

Maceroni, C. and Ribas, I.: 2007, in IAU Symposium 240: Binary Stars as Critical Tools $\&$ Tests in Contemporary Astrophysics, pp 258-260

Machida, M. N., Matsumoto, T., Hanawa, T., and Tomisaka, K.: 2005, MNRAS $\mathbf{3 6 2}, 382$

Mardling, R. A.: 2007, astro-ph/0706.0224

Margon, B.: 1984, ARA\&A 22, 507

Marilli, E., Frasca, A., Covino, E., Alcalá, J. M., Catalano, S., Fernández, M., Arellano Ferro, A., Rubio-Herrera, E., and Spezzi, L.: 2007, A $\& A$ 463, 1081

Martynov, D. I. and Khaliullin, K. F.: 1980, ApSS 71, 147

Mathieu, R. D., Duquennoy, A., Latham, D. W., Mayor, M., Mermilliod, T., and Mazeh, J. C.: 1992, in A. Duquennoy and M. Mayor (eds.), Binaries as Tracers of Stellar Formation. Proceedings of a Workshop held in Bettmeralp, 
Switzerland, Sept. 1991, in honor of Dr. Roger Griffin; Cambridge University Press, p. 278

Mathieu, R. D., Latham, D. W., and Griffin, R. F.: 1990, AJ 100, 1859

Mathieu, R. D. and Mazeh, T.: 1988, ApJ 326, 256

Mathieu, R. D., Meibom, S., and Dolan, C. J.: 2004, ApJL 602, L121

Mayor, M. and Mazeh, T.: 1987, A\&\&A 171, 157

Mayor, M. and Mermilliod, J. C.: 1984, in A. Maeder and A. Renzini (eds.), IAU Symp. 105: Observational Tests of the Stellar Evolution Theory, p. 411

Mayor, M. and Queloz, D.: 1995, Nature 378, 355

Mazeh, T.: 1990, AJ 99, 675

Mazeh, T.: 2007, in S. Udry, W. Benz, and R. von Steiger (eds.), ISSI Scientific Report Series, Vol. 6: Planetary Systems and Planets in Systems, p. 187

Mazeh, T., Krymolowski, Y., and Latham, D. W.: 1993, MNRAS 263, 775

Mazeh, T., Krymolowski, Y., and Rosenfeld, G.: 1997, ApJL 477, L103

Mazeh, T., Naef, D., Torres, G., Latham, D. W., Mayor, M., Beuzit, J.-L., Brown, T. M., Buchhave, L., Burnet, M., Carney, B. W., Charbonneau, D., Drukier, G. A., Laird, J. B., Pepe, F., Perrier, C., Queloz, D., Santos, N. C., Sivan, J.-P., Udry, S., and Zucker, S.: 2000, ApJL 532, L55

Mazeh, T. and Shaham, J.: 1976, ApJL 205, L147

Mazeh, T. and Shaham, J.: 1979, A\&A 77, 145

Mazeh, T., Tamuz, O., and North, P.: 2006, MNRAS 367, 1531

Mazeh, T., Zucker, S., Goldberg, D., Latham, D. W., Stefanik, R. P., and Carney, B. W.: 1995, ApJ 449, 909

McCluskey, Jr., G. E. and Kondo, Y.: 1971, ApESSS 10, 464

McCullough, P. R., Stys, J. E., Valenti, J. A., Fleming, S. W., Janes, K. A., and Heasley, J. N.: 2005, PASP 117, 783

McLaughlin, D. B.: 1924, ApJ 60, 22

Meibom, S. and Mathieu, R. D.: 2005, ApJ 620, 970

Meibom, S., Mathieu, R. D., and Stassun, K. G.: 2006, ApJ 653, 621

Melo, C. H. F., Covino, E., Alcalá, J. M., and Torres, G.: 2001, A\&3A 378, 898

Mermilliod, J.-C. and Mayor, M.: 1989, A\&A 219, 125

Mermilliod, J.-C. and Mayor, M.: 1990, $A \mathscr{G} A$ 237, 61

Mermilliod, J.-C. and Mayor, M.: 1999, A\&A 352, 479

Mermilliod, J.-C., Mayor, M., Andersen, J., Nordstrom, B., Lindgren, H., and Duquennoy, A.: 1989, A\&SS 79, 11

Michalska, G.: 2007, Informational Bulletin on Variable Stars 5759, 1

Michalska, G. and Pigulski, A.: 2005, A\&A 434, 89

Moorhead, A. V. and Adams, F. C.: 2007, astro-ph/0708.0335

Morris, S. L.: 1985, ApJ 295, 143

Morris, S. L. and Naftilan, S. A.: 1993, ApJ 419, 344

Naef, D., Latham, D. W., Mayor, M., Mazeh, T., Beuzit, J. L., Drukier, G. A., Perrier-Bellet, C., Queloz, D., Sivan, J. P., Torres, G., Udry, S., and Zucker, S.: 2001, $A \& A$ 375, L27

Narita, N., Enya, K., Sato, B., Ohta, Y., Winn, J. N., Suto, Y., Taruya, A., Turner, E. L., Aoki, W., Tamura, M., Yamada, T., and Yoshii, Y.: 2007, 
astro-ph/0702707

Niarchos, P. G.: 2006, ApESSS 304, 387

North, P. and Zahn, J.-P.: 2003, A\&SA 405, 677

North, P. and Zahn, J.-P.: 2004, New Astronomy Review 48, 741

Ogilvie, G. I. and Lin, D. N. C.: 2004, ApJ 610, 477

Ogilvie, G. I. and Lin, D. N. C.: 2007, ApJ 661, 1180

Orosz, J. A.: 2003, in K. van der Hucht, A. Herrero, and C. Esteban (eds.), IAU Symposium 212: A Massive Star Odyssey: From Main Sequence to Supernova, p. 365

Orosz, J. A., McClintock, J. E., Narayan, R., Bailyn, C. D., Hartman, J. D., Macri, L., Liu, J., Pietsch, W., Remillard, R. A., Shporer, A., and Mazeh, T.: 2007, Nature 449, 872

Papaloizou, J. and Lin, D. N. C.: 1984, ApJ 285, 818

Petrova, A. V. and Orlov, V. V.: 1999, AJ 117, 587

Petrova, A. V. and Orlov, V. V.: 2002, Astrophysics 45, 334

Petrova, A. V. and Orlov, V. V.: 2003, Astrophysics 46, 329

Pietsch, W., Haberl, F., Sasaki, M., Gaetz, T. J., Plucinsky, P. P., Ghavamian, P., Long, K. S., and Pannuti, T. G.: 2006, ApJ 646, 420

Pilecki, B., Fabrycky, D., and Poleski, R.: 2007, MNRAS 378, 757

Pollacco, D. L., Skillen, I., Cameron, A. C., Christian, D. J., Hellier, C., Irwin, J., Lister, T. A., Street, R. A., West, R. G., Anderson, D., Clarkson, W. I., Deeg, H., Enoch, B., Evans, A., Fitzsimmons, A., Haswell, C. A., Hodgkin, S., Horne, K., Kane, S. R., Keenan, F. P., Maxted, P. F. L., Norton, A. J., Osborne, J., Parley, N. R., Ryans, R. S. I., Smalley, B., Wheatley, P. J., and Wilson, D. M.: 2006, PASP 118, 1407

Popper, D. M.: 1980, ARAA 18, 115

Popper, D. M. and Etzel, P. B.: 1981, AJ 86, 102

Pourbaix, D., Tokovinin, A. A., Batten, A. H., Fekel, F. C., Hartkopf, W. I., Levato, H., Morell, N. I., Torres, G., and Udry, S.: 2005, Vizier Online Data Catalog 5122

Pribulla, T. and Rucinski, S. M.: 2006, AJ 131, 2986

Queloz, D., Eggenberger, A., Mayor, M., Perrier, C., Beuzit, J. L., Naef, D., Sivan, J. P., and Udry, S.: 2000, A\&A 359, L13

Rasio, F. A. and Ford, E. B.: 1996, Science 274, 954

Rasio, F. A., Tout, C. A., Lubow, S. H., and Livio, M.: 1996, ApJ 470, 1187

Rauch, T. and Werner, K.: 2003, A\&3A 400, 271

Reisenberger, M. P. and Guinan, E. F.: 1989, AJ 97, 216

Rieutord, M. and Zahn, J.-P.: 1997, ApJ 474, 760

Roberts, W. J.: 1974, ApJ 187, 575

Rossiter, R. A.: 1924, ApJ 60, 15

Russell, H. N.: 1928, MNRAS 88, 641

Sasselov, D. D.: 2003, ApJ 596, 1327

Savonije, G. J. and Papaloizou, J. C. B.: 1983, MNRAS 203, 581

Savonije, G. J. and Papaloizou, J. C. B.: 1984, MNRAS 207, 685

Savonije, G. J. and Witte, M. G.: 2002, A\&A 386, 211 
Scargle, J. D.: 1982, ApJ 263, 835

Schreier, E., Levinson, R., Gursky, H., Kellogg, E., Tananbaum, H., and Giacconi, R.: 1972, ApJL 172, L79

Seager, S., Richardson, L. J., Hansen, B. M. S., Menou, K., Cho, J. Y.-K., and Deming, D.: 2005, ApJ 632, 1122

Shakura, N. I.: 1985, Soviet Astronomy Letters 11, 224

Shporer, A., Hartman, J., Mazeh, T., and Pietsch, W.: 2007, A $\mathscr{G} A$ 462, 1091

Shu, F., Najita, J., Ostriker, E., Wilkin, F., Ruden, S., and Lizano, S.: 1994, ApJ 429, 781

Snowden, M. S. and Koch, R. H.: 1969, ApJ 156, 667

Stassun, K. G., Mathieu, R. D., Mazeh, T., and Vrba, F. J.: 1999, AJ 117, 2941

Stauffer, J. R., Hartmann, L., Soderblom, D. R., and Burnham, N.: 1984, ApJ 280, 202

Stauffer, J. R., Schild, R. A., Baliunas, S. L., and Africano, J. L.: 1987, PASP 99, 471

Sterne, T. E.: 1939a, MNRAS 99, 451

Sterne, T. E.: 1939b, MNRAS 99, 662

Sterne, T. E.: 1939c, MNRAS 99, 670

Strauss, M. A. and LSST Collaboration: 2006, Vol. 38 of Bulletin of the American Astronomical Society, p. 1017

Sutantyo, W.: 1976, A\&\&A 52, 141

Tamuz, O., Mazeh, T., and North, P.: 2006, MNRAS 367, 1521

Tananbaum, H., Gursky, H., Kellogg, E. M., Levinson, R., Schreier, E., and Giacconi, R.: 1972, ApJL 174, L143

Tassoul, J.-L.: 1995, ApJ 444, 338

Tassoul, M. and Tassoul, J.-L.: 1997, ApJ 481, 363

Terquem, C., Papaloizou, J. C. B., Nelson, R. P., and Lin, D. N. C.: 1998, ApJ $\mathbf{5 0 2}, 788$

Thommes, E. W. and Lissauer, J. J.: 2003, ApJ 597, 566

Tokovinin, A. A.: 1997, $A \& B S$ 124, 75

Tokovinin, A. A.: 2004, Vol. 21 of Revista Mexicana de Astronomia y Astrofisica Conference Series, p. 7

Tokovinin, A. A., Balega, Y. Y., Pluzhnik, E. A., Shatsky, N. I., Gorynya, N. A., and Weigelt, G.: 2003, A\&A 409, 245

Tokovinin, A. A. and Smekhov, M. G.: 2002, A\&SA 382, 118

Tokovinin, A. A., Thomas, S., Sterzik, M., and Udry, S.: 2006, A\&SA 450, 681

Trilling, D. E.: 2000, ApJL 537, L61

Trilling, D. E., Benz, W., Guillot, T., Lunine, J. I., Hubbard, W. B., and Burrows, A.: 1998, ApJ 500, 428

Udalski, A., Kubiak, M., and Szymanski, M.: 1997, Acta Astronomica 47, 319

Udalski, A., Soszynski, I., Szymanski, M., Kubiak, M., Pietrzynski, G., Wozniak, P., and Zebrun, K.: 1998, Acta Astronomica 48, 563

Udry, S., Jorissen, A., Mayor, M., and Van Eck, S.: 1998, A $\mathscr{E} A S$ 131, 25

van Kerkwijk, M. H., Kaspi, V. M., Klemola, A. R., Kulkarni, S. R., Lyne, A. G., and Van Buren, D.: 2000, ApJ 529, 428 
Verbunt, F.: 1994, $A \mathscr{E} A$ 285, L21

Verbunt, F. and Phinney, E. S.: 1995, A\&A 296, 709

Walker, G. A. H., Croll, B., Kuschnig, R., Walker, A., Rucinski, S. M., Matthews, J. M., Guenther, D. B., Moffat, A. F. J., Sasselov, D., and Weiss, W. W.: 2007, ApJ 659, 1611

Walker, M. F. and Bell, M.: 1980, ApJ 237, 89

Ward, W. R. and Hahn, J. M.: 1994, Icarus 110, 95

Weidenschilling, S. J. and Marzari, F.: 1996, Nature 384, 619

Weinberg, S.: 1972, Gravitation and Cosmology: Principles and Applications of the General Theory of Relativity, Wiley-VCH

Wheeler, J. C., McKee, C. F., and Lecar, M.: 1974, ApJL 192, L71

Winn, J. N. and Holman, M. J.: 2005, ApJL 628, L159

Winn, J. N., Holman, M. J., Johnson, J. A., Stanek, K. Z., and Garnavich, P. M.: 2004, ApJL 603, L45

Winn, J. N., Johnson, J. A., Marcy, G. W., Butler, R. P., Vogt, S. S., Henry, G. W., Roussanova, A., Holman, M. J., Enya, K., Narita, N., Suto, Y., and Turner, E. L.: 2006, ApJL 653, L69

Winn, J. N., Johnson, J. A., Peek, K. M. G., Marcy, G. W., Bakos, G. Á., Enya, K., Narita, N., Suto, Y., Turner, E. L., and Vogt, S. S.: 2007, ApJL 665, L167

Winn, J. N., Noyes, R. W., Holman, M. J., Charbonneau, D., Ohta, Y., Taruya, A., Suto, Y., Narita, N., Turner, E. L., Johnson, J. A., Marcy, G. W., Butler, R. P., and Vogt, S. S.: 2005, ApJ 631, 1215

Witte, M. G. and Savonije, G. J.: 2002, $A \xi A$ 386, 222

Wood, F. B., Parker Oliver, J., and Koch, R. H.: 1980, A finding list for observers of interacting binary stars, Publ. Dept. of Astronomy, Univ. of Florida - Publ. Univ. of Pennsylvania, Astron. Ser.: Univ. of Pennsylvania Press, 5th ed.

Worek, T. F.: 1996, PASP 108, 962

Wyithe, J. S. B. and Wilson, R. E.: 2001, ApJ 559, 260

Wyrzykowski, L., Udalski, A., Kubiak, M., Szymanski, M. K., Zebrun, K., Soszynski, I., Wozniak, P. R., Pietrzynski, G., and Szewczyk, O.: 2004, Acta Astronomica 54, 1

Zahn, J. P.: 1966, Annales d'Astrophysique 29, 313

Zahn, J.-P.: 1975, A\&A 41, 329

Zahn, J.-P.: 1977, A\&A 57, 383

Zahn, J.-P.: 1989, A\&A 220, 112

Zahn, J.-P. and Bouchet, L.: 1989, A\&A 223, 112

Zhang, L.-Y. and Gu, S.-H.: 2007, $A \mathscr{E A}$ 471, 219

Zhou, J.-L. and Lin, D. N. C.: 2007, ApJ 666, 447

Zucker, S., Mazeh, T., Santos, N. C., Udry, S., and Mayor, M.: 2003, A 63 A 404, 775

Zucker, S., Mazeh, T., Santos, N. C., Udry, S., and Mayor, M.: 2004, A\&̇A 426, 695 